\title{
Transgressive segregation for salt tolerance in rice due to physiological coupling and uncoupling and genetic network rewiring
}

\section{Short title: Molecular basis of transgressive segregation}

\author{
Isaiah C.M. Pabuayon ${ }^{1}$, Ai Kitazumi ${ }^{1}$, Kevin R. Cushman ${ }^{1}$, Rakesh Kumar Singh ${ }^{2 \# a,}$ \\ Glenn B. Gregorio ${ }^{2 \# b}$, Balpreet Dhatt ${ }^{3}$, Masoud Zabet-Moghaddam ${ }^{4}$, \\ Harkamal Walia ${ }^{3}$, Benildo G. de los Reyes ${ }^{1^{*}}$
}

${ }^{1}$ Department of Plant and Soil Science, Texas Tech University, Lubbock, TX USA ${ }^{2}$ International Rice Research Institute, Los Baños, Laguna, Philippines

${ }^{3}$ Department of Agronomy and Horticulture, University of Nebraska, Lincoln, NE USA

${ }^{4}$ Center for Biotechnology and Genomics, Texas Tech University, Lubbock, TX USA

${ }^{\# a}$ Current address: International Center for Biosaline Agriculture, Dubai, United Arab Emirates

${ }^{\# b}$ Current address: Southeast Asian Regional Center for Graduate Studies in Agriculture, Los Baños, Laguna, Philippines

*Author for correspondence: benildo.reyes@ttu.edu; 806-834-6421

Abbreviations: QTL, quantitative trait loci; RIL, recombinant inbred line; SES, standard evaluation score; ELI, electrolyte leakage index; LP, lipid peroxidation; POX, total peroxidase activity; APS, aggregate phenotypic score; NPT, new plant-type; PCA, principal component analysis; SA, salicylic acid; JA, jasmonic acid; TCA cycle, tricarboxylic acid cycle

The author responsible for distribution of materials integral to the findings presented in this article in accordance with the policy described in the Instructions for Authors (www.plantcell.org) is: Benildo G. de los Reyes (benildo.reyes@ttu.edu). 


\section{Abstract}

Transgressive segregation is common in plant breeding populations, where a small minority of recombinants are outliers relative to parental phenotypes. While this phenomenon has been attributed to complementation and epistatic effects, the physiological, biochemical, and molecular bases have not been fully illuminated. By systems-level scrutiny of the IR29 x Pokkali recombinant inbred population of rice, we addressed the hypothesis that novel salt tolerance phenotypes are created by positive or negative coupling or uncoupling effects and novel regulatory networks. Hyperspectral profiling distinguished the transgressive individuals in terms of stress penalty to growth. Non-parental network signatures that led to either optimal or non-optimal integration of developmental with stress-related mechanisms were evident at the macro-physiological, biochemical, metabolic, and transcriptomic levels. The large positive net gain in supertolerant progeny was due to ideal complementation of beneficial traits, while shedding antagonistic traits. Super-sensitivity was explained by the stacking of multiple antagonistic traits and loss of major beneficial traits. The mechanisms elucidated in this study are consistent with the Omnigenic Theory, emphasizing the synergy or lack

44 thereof between core and peripheral components. This study supports a breeding

45 paradigm based on genomic modeling to create the novel adaptive phenotypes for the crops of the $21^{\text {st }}$ century. 


\section{Introduction}

Plant breeding during the Green Revolution of the 1960's successfully created the modern crop cultivars with superior yields under well managed environments with ideal water and nutrient conditions. Because of climate change and depletion of natural resources, the new challenges to the $21^{\text {st }}$ century plant breeding have become even grander (Khush, 2001, 2005; Pingali, 2012). Aided by high-throughput genotyping, marker-assisted selection, transgenic technologies, and by the emerging paradigms of gene editing and genomic selection, plant breeding has entered a new era that calls for the ability to further fine-tune genotype $x$ environment $(G \times E)$ interaction to create novel phenotypes that were not fully addressed by the earlier breeding paradigms (de los Reyes, 2019).

Developing the next generation of crop cultivars with minimal penalty to yield under marginalized environments has become the new overarching goal of plant breeding. Key to this goal is the creation of new genetic variants and novel phenotypes. To create such novel phenotypes, a non-reductionist approach is important in considering several fundamental questions. Has plant breeding fully exhausted the potential of exotic germplasm to create novel phenotypes that are more relevant today than prior to the green revolution? With genomic biology, there is a strong optimism that precise utilization of the combining potentials of exotic germplasm could lead to further improvements in complex 'omnigenic' traits that define yield potential under marginal environments (Boyle et al., 2017). Are further genetic gains on top of what was achieved during the green revolution still possible? If so, how can the power of genomic biology be used for additional genetic gains? Genomics-enabled selection has allowed breeders to genotype each individual in breeding populations and evaluate genetic gains through the inheritance of quantitative trait loci (QTL; Li et al., 2005; Lippman et al., 2007; Thomson et al., 2010). The paradigm of QTL stacking promises to optimize

74 QTL combinations to exploit the additive and non-additive potentials of both parents for maximal gains (deVicente and Tanksley, 1993; Eshed and Zamir, 1995; Tanksley and Nelson, 1996; Ashikari and Matsuoka, 2006; Kumar et al., 2018; Sandhu et al., 2018). Both questions should inspire critical thinking to combine the classic phenomena in plant breeding with genomic biology towards the creation of novel phenotypes.

The author responsible for distribution of materials integral to the findings presented in this article in accordance with the policy described in the Instructions for Authors (www.plantcell.org) is: Benildo G. de los Reyes (benildo.reyes@ttu.edu). 
79 Transgressive segregation is one good example of such phenomena. It was proposed

80 that transgressive phenotypes in breeding populations are similar to the genetic

81 novelties in natural population of hybrids that serve as foundation for adaptive speciation (Rieseberg et al., 1996; Dickinson et al., 2003; Rieseberg et al., 2003; de los Reyes, 2019). Transgressive segregation is characterized by heritable variation across the progenies of genetically divergent parents where a small minority are beyond the parental range and thus differs explicitly from heterosis (Bomblies and Weigel, 2007; Lippman and Zamir, 2007; Birchler et al., 2010). Inspired by evolutionary biology, a classic phenomenon such as transgressive segregation should be re-envisioned as a possible means to achieve further genetic gains in adaptive potentials for the $21^{\text {st }}$ century crop varieties.

Epistatic interactions of parental alleles and complementation effects of additive alleles are considered to be the major causes of superior or inferior attributes of transgressive segregants (Dittrich-Reed and Fitzpatrick, 2013). The recently proposed Omnigenic Theory defines quantitative traits in terms of both additive and non-additive contributions of few large-effect core loci and hundreds of minute-effect peripheral loci across the genome (Boyle et al., 2017). We further hypothesized that the superiority or inferiority of transgressive segregants are due to ideal coupling/uncoupling or non-ideal coupling/uncoupling, respectively, of the various compatible and incompatible biochemical and developmental attributes from each parent encoded by the core and peripheral loci. Such mechanisms lead to either physiological gain or physiological drag in certain individuals, determining positive or negative net gain (de los Reyes, 2019). Because of the many potential attributes that should be affected by such coupling and uncoupling mechanisms, it would take many generations of genetic recombination and genome reshuffling to observe the physiological gain or drags on a relatively small number of individuals in a segregating population.

To test the physiological coupling and uncoupling and network rewiring hypotheses, we employed a multi-tier approach to examine a well characterized recombinant inbred population of rice for salt tolerance that was developed by the International Rice Research Institute (IRRI) in the 1990's from the parental cultivars IR29 (Xian/Indica; salt-sensitive) and Pokkali (Aus; salt-tolerant) (Bonilla et al., 2002; 
110 Gregorio et al., 2002; Walia et al., 2005; Singh et al., 2007; Thomson et al., 2010).

111 Pokkali is a photoperiod-sensitive landrace historically used as donor of salt tolerance in 112 rice breeding programs in India (Moeljopawiro and Ikehashi, 1981; Sahi et al., 2006).

113 We were particularly interested in exploring the full combining potential of this donor 114 with a salt-sensitive but high-yielding Xian/Indica cultivar (IR29) beyond what has been 115 shown by QTL mapping. QTL mapping conducted on this population identified the Saltol 116 on chromosome-1 of Pokkali to be responsible for much of phenotypic variance for salt 117 stress defenses at the seedling stage. Saltol included genes involved in the regulation 118 of $\mathrm{Na}^{+}$uptake and accumulation in the shoots (Ren et al., 2005; Thomson et al., 2010). 119 The recombinant inbred line $\left(\mathrm{RIL}, \mathrm{F}_{8}\right)$ FL478 has served as the non-photoperiod 120 sensitive donor of Saltol to improve the seedling-stage salt tolerance of Xian/Indica rice 121 cultivars (Huyen et al., 2012; Islam et al., 2012; Huyen et al., 2013; Chattopadhyay et 122 al., 2014; Bimpong et al., 2016; Waziri et al., 2016; Babu et al., 2017).

123 We describe here the results of a multi-tier macro-physiological, biochemical, and 124 molecular profiling of the IR29 x Pokkali RILs at the population and individual levels. We 125 scrutinized specific individuals representing each phenotypic class by integrating multi126 level 'omics' datasets. With this approach, we were able to identify clear transgressive 127 segregants and provide strong support to the coupling and uncoupling hypothesis by 128 revealing the hidden potentials of the salt-sensitive Xian/Indica parent IR29 towards 129 positive complementation with Saltol and other genes from Pokkali. We also revealed 130 the potential of Pokkali as a source of physiological drags against other minor effect 131 genes. This study also provides direct evidence that transgressive traits are created by 132 network rewiring as an outcome of recombination. 
Results

\section{Evaluation of the IR29 x Pokkali RILs under severe salt stress}

During the fine-mapping of Saltol, the IR29 x Pokkali core mapping population $\left(F_{2}, F_{8}\right.$-RILs of 126 individuals) was evaluated at IRRI at the seedling stage (V1 to V5) under $\mathrm{EC}=9 \mathrm{dS} / \mathrm{m}$ for $\mathrm{Na}^{+}$exclusion potential, with shoot and root $\mathrm{Na}^{+} / \mathrm{K}^{+}$and Standard Evaluation Score (SES) as main criteria (Counce et al., 2000). The SES ranges from 1 to 9 , with 1 representing the highest level of tolerance. IRRI selected 64 $\mathrm{F}_{8}$-RILs (representative group) spanning the range of salt tolerance across the population. Along with the parents IR29 and Pokkali, and an additional salt-sensitive check IR64, we conducted a time-course $(0,24,48,72,144 \mathrm{hr})$ evaluation of salt tolerance across the representative groups at tillering stage (V4 to V12). A more severe salt stress $(E C=12 \mathrm{dS} / \mathrm{m})$ was used to further push the limits of phenotypic potentials that were not revealed by the earlier milder treatments. Using SES as initial criterion, we expected to identify outlier RILs that perform worse than IR29 or better than Pokkali at V4 to V12. Results revealed slightly different ranking with clear outliers not identified at V1 to V5 (Supplemental Data Set 1). From these, we established the minimal comparative panel, consisting of thirty seven (37) RILs spanning the full range of tolerance revealed at EC $=9 \mathrm{dS} / \mathrm{m}$ (sensitive = IR29, FL454; tolerant = Pokkali, FL478), and the outliers revealed at EC $=12 \mathrm{dS} / \mathrm{m}$, consisting of the super-sensitive FL499 and super-tolerant FL510 (Table 1, Figure 1A).

The minimal comparative panel was further profiled for a set of physiomorphometric parameters including electrolyte leakage index (ELI), cellular ion concentration, lipid peroxidation (LP) as a measure of cellular membrane injury, total peroxidase activity (POX) as measure of ROS scavenging capacity, shoot biomass, and stomatal conductance (Figure 1B). Only mild correlation with SES was established (Supplemental Table 1), including POX at the point (144 hr) of maximum injury $\left(\mathrm{r}^{2}=\right.$ $0.17)$, shoot $\mathrm{Na}^{+} / \mathrm{K}^{+}\left(r^{2}=0.15\right)$, and POX stress/control ratio $\left(r^{2}=0.11\right)$. Collectively, the physio-morphometric parameters did not correlate strongly with SES.

The tolerant FL478 carrying an introgression for Saltol allele of Pokkali was as 164 tolerant as Pokkali under both $\mathrm{EC}=9 \mathrm{dS} / \mathrm{m}$ and $\mathrm{EC}=12 \mathrm{dS} / \mathrm{m}$ (Table 1, Figure 1A; 165 (Thomson et al., 2010). Other RILs with homozygous introgression for Pokkali Saltol 


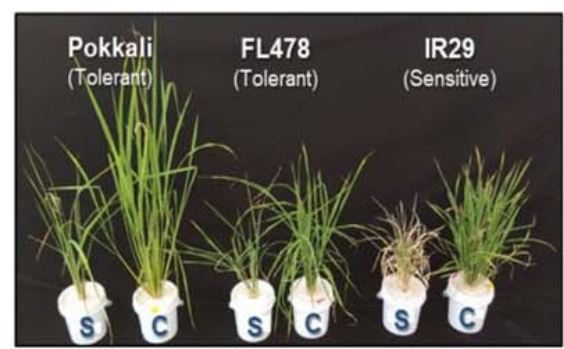

FL499

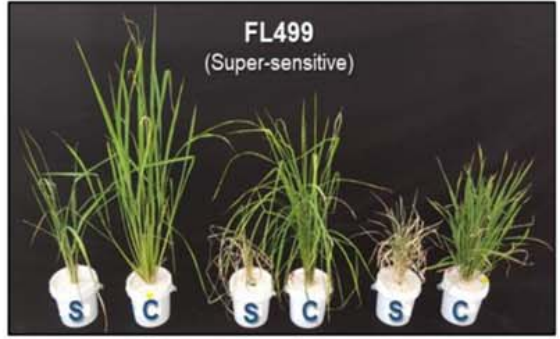

FL510

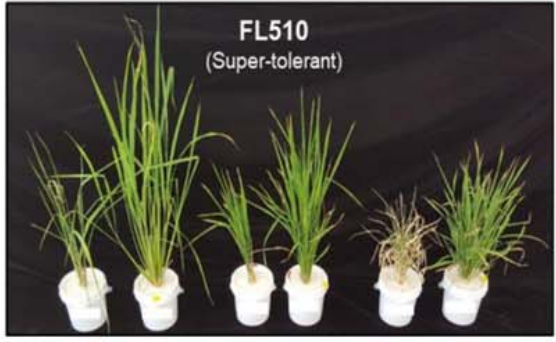

\section{A}

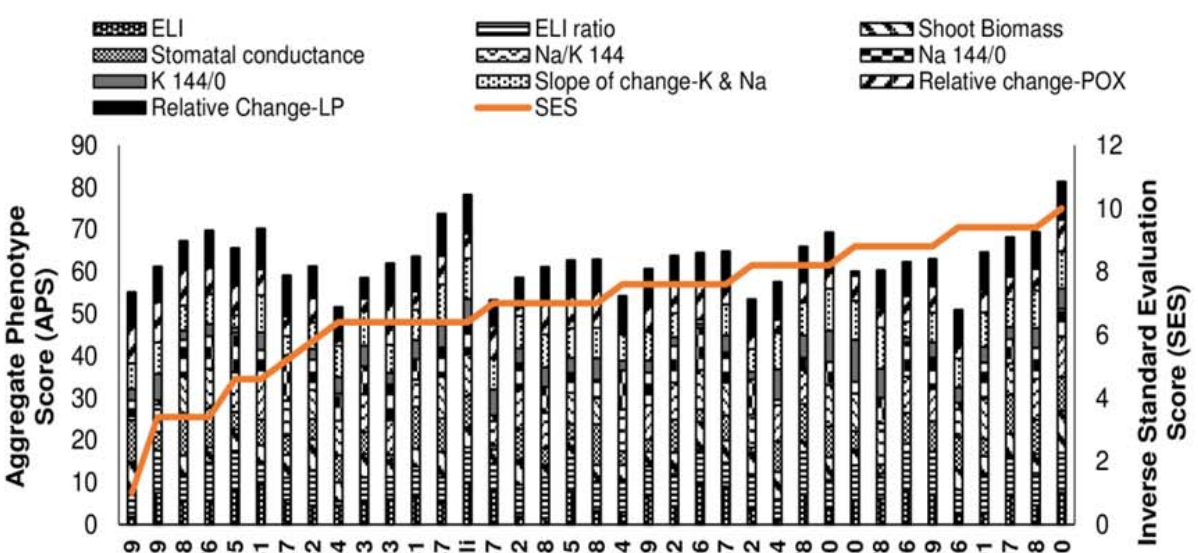

B 8.

Figure 1. Salt tolerance at V4 to V8 stage across the minimal comparative panel representing the full phenotypic range at EC= $9 \mathrm{dS} / \mathrm{m}$ (IRRI) and EC= $12 \mathrm{dS} / \mathrm{m}$ (Texas). (A) Comparison of plant health in control (C) and stress (S) experiments after seven days (144 hrs) at EC=12 dS/m. The outliers FL510 (super-tolerant) and FL499 (super-sensitive) are highlighted compared to tolerant parent Pokkali (Saltol donor), sensitive parent IR29, and tolerant sibling FL478 (see Table 1). Differences in injury and growth were evident particularly between the transgressive FL510 and FL499. (B) Individual physio-morphometric scores were normalized and combined as Aggregate Phenotypic Score (APS), which assumes equal weights of each parameter that includes Electrolyte Leakage Index (ELI) and its ratio (ELI ratio) at first injury (72 hr) and maximum injury (144 hr), shoot biomass ratio (stress/control), stomatal conductance ratio (stress/control), $\mathrm{Na}^{+} / \mathrm{K}^{+}$at maximum injury $(\mathrm{Na} / \mathrm{K}$ $144 / 0), \mathrm{Na}^{+}$ratio at maximum injury ( $\left.\mathrm{Na} 144 / 0\right), \mathrm{K}^{+}$ratio at maximum injury (K 144/0), slope of $\mathrm{K}^{+}$and $\mathrm{Na}^{+}$ changes (control/stress), change in peroxidase activity (change-POX; stress/control), and change in lipid peroxidation (change-LP; stress/control). APS was plotted against the inverse of SES for direct proportionality. 
confirming the biological significance of the blind SES ranking at EC $=12 \mathrm{dS} / \mathrm{m}$. As an outlier, the super-tolerant FL510 had the best SES and highest aggregate phenotypic score (APS) across the population, significantly outperforming the tolerant Pokkali and FL478. While the individual scores of FL510 were not always the best, none of them were below the population means, implying the additive effects of many minutely contributing parameters to its overall potential. FL478 tended to have very good scores in some parameters but poor scores in others, which appeared to cause a penalty to the net phenotypic score. Pokkali had a very good APS, but its SES was inferior to FL510 (Figure 1B).

The sensitive parent IR29 along with about $30 \%$ of RILs including FL454 (sensitive) and FL499 (super-sensitive) were the worst performers based on SES alone (Supplemental Data Set 1; Table 1). FL499 was severely injured with the worst scores for most parameters especially for ELI and $\mathrm{Na}^{+} / \mathrm{K}^{+}$. The trends in APS showed that the poor ranking of FL499 was due to poor scores in the majority but not all parameters (Figure 1B). Integration of all physio-morphometric parameters into an aggregate score (APS) indicate the various permutations by which different parental attributes could be combined in the progenies either optimally or non-optimally.

\section{Relatedness among RILs based on physio-morphometric matrix}

Based on few parameters where the sensitive parent IR29 had significantly better scores than the tolerant parent Pokkali, we hypothesized that some of IR29-derived properties may be contributing to tolerance potential when combined with other complementary properties from Pokkali. To address this hypothesis, we used the total matrix to measure the similarities across RILs through neighbor-joining dendrograms. The first measure established global relationships based on all contributing factors. The second was based only on $\mathrm{Na}^{+}$exclusion components $\left(\mathrm{Na}^{+}\right.$content, $\mathrm{K}^{+}$content, $\mathrm{Na}^{+} / \mathrm{K}^{+}$, ELI) to assess the contribution of Saltol (Table 1).

The full-matrix dendrogram illustrates the overall range of similarities (Figure 2A), where the super-sensitive FL499 formed the most distant clade. The other poor RILs (e.g., IR29, FL454) formed distinct clades from the better RILs (e.g., Pokkali, FL478, FL510). The super-tolerant FL510 also formed the most distant sub-clade among the 

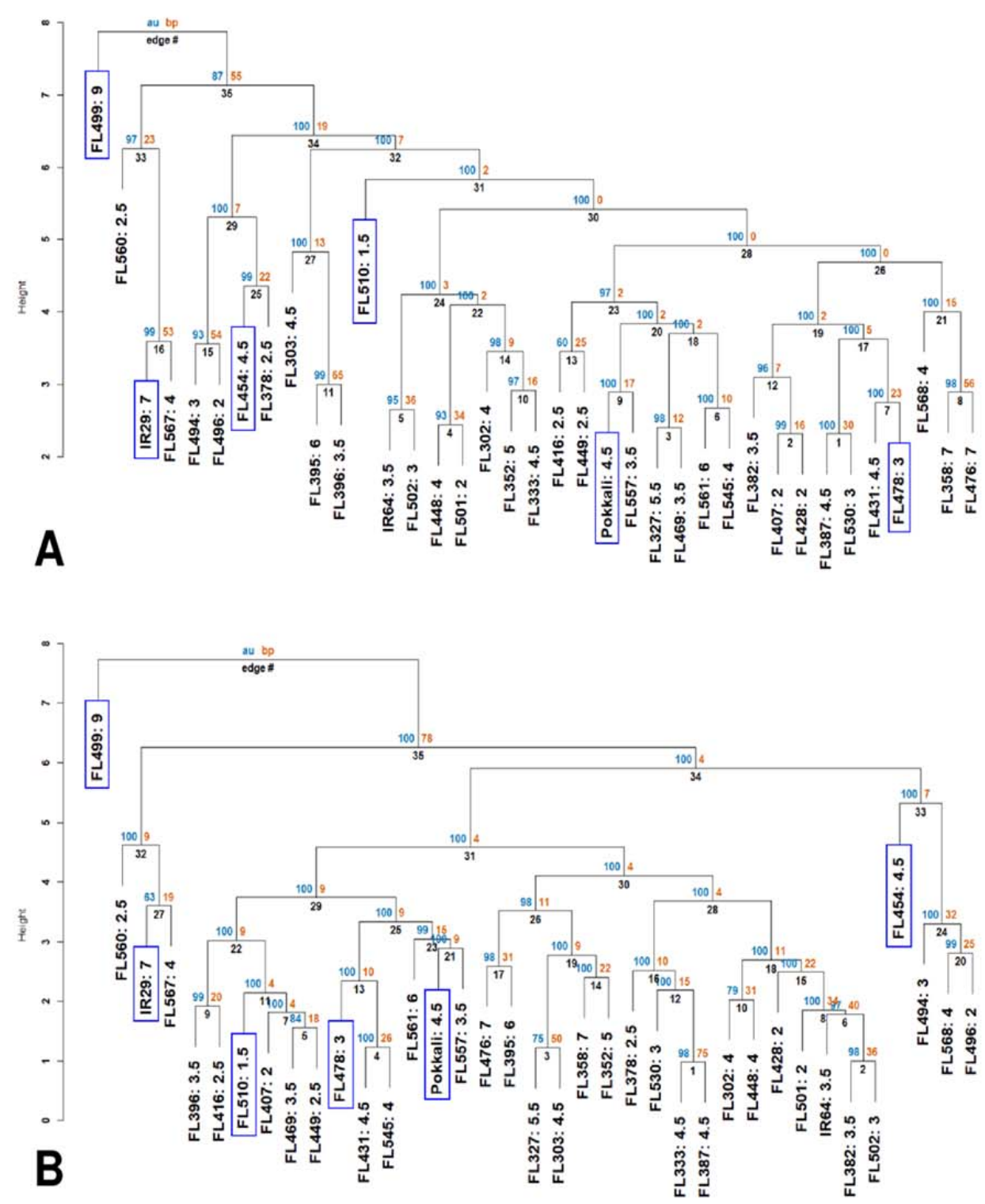

Figure 2. Neighbor-joining dendrograms showing two layers of similarities based on the phenotypic matrix (APS, SES). Each genotype in the dendrogram is suffixed with their SES. The individual genotypes (parents, RILs) that were investigated to understand the physiological mechanisms are highlighted in blue boxes. AU (blue) refers to the adjusted P-value of clustering, and BP (orange) refers to the bootstrapping value of 'pvclust' package. (A) Similarities based on the entire matrix (components of APS + SES). Dendrogram shows the poor genotypes represented by IR29, FL454, and FL499 forming distinct clades from the good genotypes represented by Pokkali, FL478, and FL510. (B) Similarities based on APS components relevant to $\mathrm{Na}^{+}$sequestration ( $\mathrm{ELI}, \mathrm{Na}^{+}$and $\mathrm{K}^{+}$contents at control and $144 \mathrm{hr}, \mathrm{Na}^{+} / \mathrm{K}^{+}$ratio). Dendrogram shows the tendency for individuals carrying the Pokkali Saltol allele to cluster together within one large clade. In both (A) and (B), the super-sensitive FL499 had the earliest divergence. 
mostly good RILs also included few inferior RILs, suggesting that even the poor RILs shared some attributes with their better siblings.

Saltol effects are primarily associated with $\mathrm{Na}^{+}$exclusion. The dendrogram based on salt exclusion components revealed even more meaningful groupings (Figure 2B).

203 The clear outliers were the inferior RILs (e.g., IR29, FL454, FL499) that are negative for

204 Saltol (Table 1). The large clade consisting of mostly good RILs is divided into two sub205 clades. The first is comprised of RILs that are positive for Saltol (tolerant Pokkali and 206 FL478, and super-tolerant FL510), with Pokkali and FL478 being more closely related to 207 each other than to FL510. The second is comprised of mostly (13 out of 17) good and 208 moderate RILs that are positive for Saltol, indicating that the similarities among the good 209 RILs are largely due to Saltol. However, such similarities excluded the contributions of 210 other properties in the full matrix, hence without the contributions of other factors in the 211 genetic background.

\section{Real-time profiling of growth and physiological potentials}

We used the representative phenotypic classes comprised of the sensitive parent (IR29), tolerant parent (Pokkali), sensitive RIL (FL454), tolerant RIL (FL478), supersensitive RIL (FL499), and super-tolerant RIL (FL510) to track plant growth in real-time with the LemnaTec imaging system for a period of 18 days from V4 to V12. RGB imaging was used to determine the projected shoot area (PSA) and plant height, which are correlated with plant biomass (Campbell et al., 2015). Plants were stressed at EC = $9 \mathrm{dS} / \mathrm{m}$ to remove potential bias against the sensitive classes given that $\mathrm{EC}=12 \mathrm{dS} / \mathrm{m}$ was nearly lethal for FL499. Nevertheless, EC = $9 \mathrm{dS} / \mathrm{m}$ was strong enough to

222 differentiate the potentials across the panel.

The image-based growth curves based on daily measurements of plant area and

224 height showed linear growth patterns over time (Figure 3A). However, major differences 225 even in the control were evident in terms of inherent growth potentials. Growth rates in 226 Pokkali, FL454, and FL499 were much higher compared to IR29, FL478 and FL510 227 even in the control. In the RILs, the growth potentials appeared to be due to the 228 combined effects of parental attributes. For instance, the fast-growing salt-sensitive 229 RILs (FL454, FL499) were more like the salt-tolerant parent Pokkali in terms of plant 

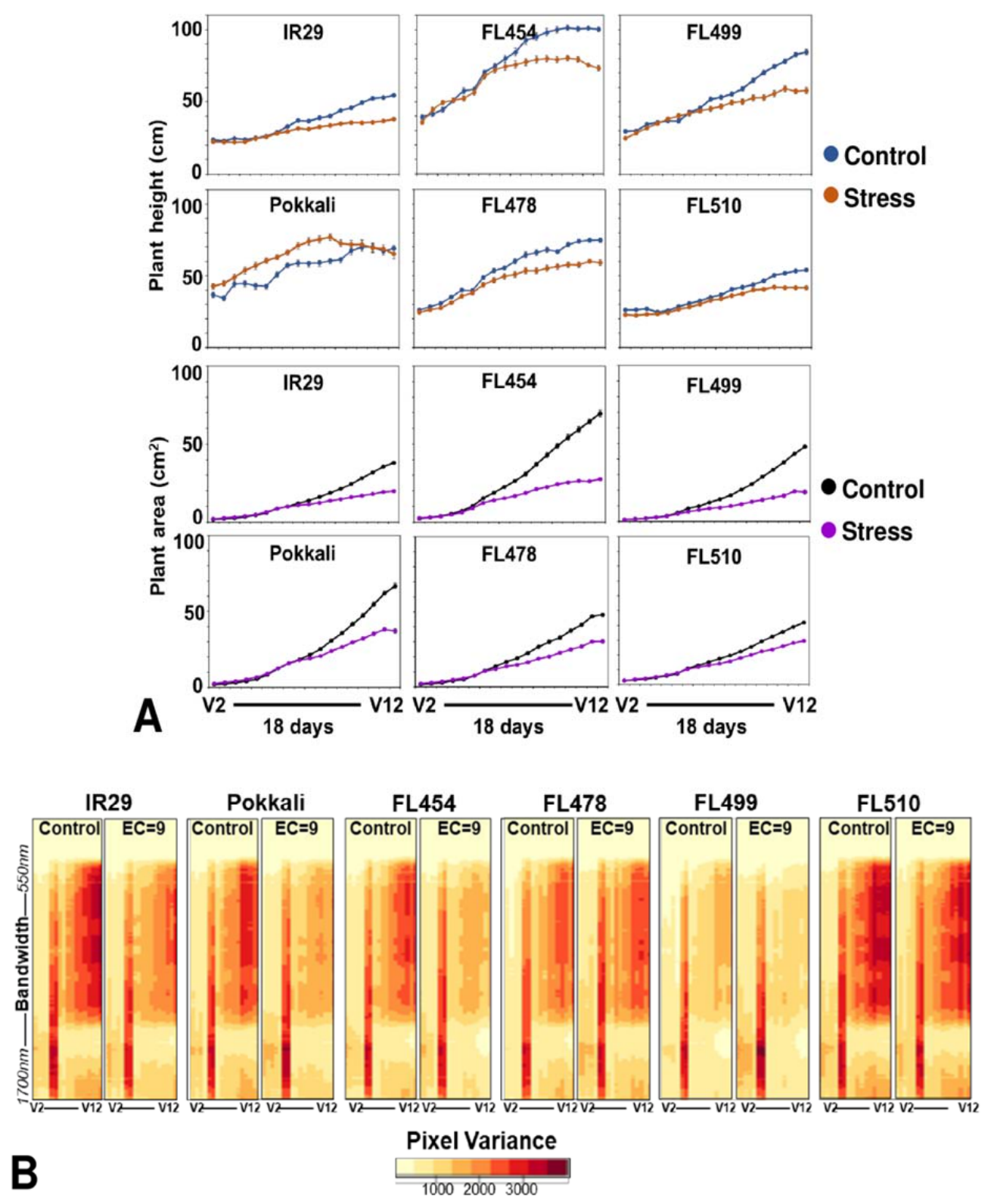

Figure 3. Real-time growth profiling of the sensitive parent IR29, tolerant parent Pokkali, sensitive RIL FL454, tolerant RIL FL478, super-sensitive RIL FL499, and super-tolerant RIL FL510 at EC= 9 $\mathrm{dS} / \mathrm{m}$. (A) Growth curves as a function of changes in plant area and plant height during the 18-day period of stress, calculated based on pixels captured by RGB and hyperspectral camera. Note the time of forking between the control and stress curves and the angle of the fork, reflective of growth plateauing. (B) Variation in hyperspectral variance at 243 wavelength bands ranging from $550 \mathrm{~nm}$ to $1700 \mathrm{~nm}$. Heat maps of hyperspectral variances are potential indicators of overall plant health and stress injuries. The overall patterns in hyperspectral variances mirror the patterns revealed by the growth curve analysis. 
231 (FL478, FL510) were more similar in overall growth pattern to the salt-sensitive parent

232 IR29. The high-yielding improved Xian/Indica variety IR29 has short stature with profuse 233 tillering, while the Aus landrace Pokkali has tall stature, darker green leaves, and less

234 tillering. FL478 has the same Xian/Indica plant-type as IR29, while FL510 has distinct 235 morphology (Figure 1A). It is intermediate in height, with thick, dark green, and erect 236 leaves and stems, similar to the new plant-type (NPT) bred at IRRI during the 1990's 237 (Peng et al., 1994; Peng et al., 2008).

Growth curves indicate that stress penalty was common across all genotypes

239 (Figure 3A). However, the magnitude of penalty varied significantly regardless of the 240 inherent variation in growth potentials under control condition. For instance, the timing 241 when growth retardation occurred varied with clear correlation to salt tolerance. Plants 242 under control and stress grew at similar rates before stress. While growth retardation 243 based on projected shoot area (PSA) and height was detectable as early as seven (7) 244 days after stress, variation across genotypes was evident based on the timing of the 245 forking between the control and stress curves, which determined the magnitude of 246 growth plateauing (i.e., fork angle) in real-time (Figure 3A). Growth penalty was most 247 severe in the salt-sensitive IR29, FL454 and FL499 relative to their respective controls.

248 This was also supported by few other parameters such as tiller reduction, plant 249 biomass, and symptoms of leaf injury. Tillering of Pokkali and FL478 were also 250 negatively affected but in much lesser magnitude than IR29, FL454, and FL499. The 251 super-tolerant FL510 had the least penalty based on the degree of tiller reduction and 252 timing and angle of forking (Figure 3A). The PSA ratio (stress/control) also indicate 253 difference in tolerance based on growth maintenance (Supplemental Figure 1). Tiller 254 reduction was evident from decreasing PSA ratio in all genotypes. However, FL510 and 255 FL478 stayed close to a ratio of 1 , indicating much less damage.

256 To further substantiate the observed variation in growth penalty, we examined 257 the hyperspectral profiles across the growth window as potential indicator of plant health 258 and vigor. Hyperspectral profiles were based on image captured at 243 wavelength 259 bands ranging from $550 \mathrm{~nm}$ to $1700 \mathrm{~nm}$, with each band evenly separated by $4.7 \mathrm{~nm}$. 260 Image variance plots described the spread of pixel intensities as indicators of how much 261 physical changes including wilting, drying, yellowing or necrosis occurred during stress. 
262 Differences in growth across genotypes (Figure 3A) were generally consistent with the 263 trends in hyperspectral profiles (Figure 3B). Reduction in image variances was quite 264 apparent in IR29 and FL454 but only minimal in FL510 and FL478, indicative of lesser 265 injuries. These genotypes had more stable profiles throughout the stress period. FL499 266 had lower image variances even under control, suggestive of less than optimal growth 267 that was further exacerbated by stress. Interestingly, the hyperspectral profiles of the 268 super-tolerant FL510 in both control and stress were quite similar to the high-yielding 269 parent IR29 under control. This suggests a positive attribute acquired by FL510 from 270 IR29, which translated into net gains in combination with other defense-related traits 271 inherited from Pokkali.

$\mathrm{Na}^{+} / \mathrm{K}^{+}$and proline profiles in relation to real-time growth variances

The dynamics of $\mathrm{Na}^{+}$and $\mathrm{K}^{+}$accumulation was investigated to assess how much the capacity for $\mathrm{Na}^{+}$exclusion contributed to the growth penalty in sensitive and tolerant genotypes. The $\mathrm{Na}^{+} / \mathrm{K}^{+}$ratio $\left(\mathrm{Na}^{+} / \mathrm{K}^{+}\right)$in shoots and roots were used as primary indicators of tolerance. Variation for $\mathrm{Na}^{+} / \mathrm{K}^{+}$in shoot across genotypes was significant in stress but not in control (Supplemental Figure 2A). IR29, FL454, and FL499 had significantly higher $\mathrm{Na}^{+} / \mathrm{K}^{+}$than Pokkali, FL478, and FL510. The mean $\mathrm{Na}^{+} / \mathrm{K}^{+}$ segregated the panel into tolerant (Pokkali, FL478, FL510) and sensitive (IR29, FL454, FL499) groups $(\mathrm{P}<0.05)$. Because of Saltol, Pokkali had the lowest $\mathrm{Na}^{+} / \mathrm{K}^{+}$within the tolerant group, thus the overall potentials of FL478 and FL510 may not dependent on Saltol effects alone. No significant difference in root $\mathrm{Na}^{+} / \mathrm{K}^{+}$was detected across genotypes (Supplemental Figure 2B).

Proline is known to contribute to the maintenance of cellular water potential by osmotic adjustment (Claussen, 2005; Ashraf and Foolad, 2007; Kumar et al., 2010;

287 Szabados and Savoure, 2010). The total proline content of each genotype was 288 measured after seven (7) days of salt stress, which also correspond to the initiation of 289 the forking between the control and stress growth curves (Figure 3A). Proline content 290 increased significantly $(P<0.05)$ under salt stress in IR29 and FL454 but not in other 291 genotypes (Supplemental Figure 2C), suggesting that osmotic adjustment is not a major contributing factor to the variation in growth penalties. 


\section{Metabolite profiles across the gradient of salt tolerance potential}

Shotgun profiling by LC-MS/MS was performed to compare the stress metabolite signatures across genotypes. About 7,000 unique macromolecules were identified, many of which were unique to a given genotype. A total of 217 known metabolites had differential abundances between stress and control at $\mathrm{P}<0.05$ (Supplemental Data Set 2). K-means clustering and principal component analysis (PCA) revealed multiple components that spread the entire dataset widely based on variances. FL499 had the most distinct profile compared to the other genotypes (Figure 4A). The PCA plot revealed significant overlaps among the tolerant genotypes (FL478, FL510) for the differentially abundant metabolites, with patterns completely opposite to the profiles of the parents (IR29, Pokkali) and super-sensitive FL499. These trends indicate metabolite signatures associated with salt tolerance. The super-tolerant FL510 was more similar to the sensitive parent IR29, while the tolerant FL478 was more similar to the tolerant parent Pokkali (Figure 4B). All these suggest that the improved high-yielding but saltsensitive parent IR29 has significant contributions to the overall potential of the transgressive super-tolerant FL510.

The K-means clustering of differentially abundant metabolites revealed different patterns of co-expression (Figure 4C). Clusters-1, 2 and 4, which are enriched with different types of flavonoids and other antioxidants have similar profiles in IR29 and

313 FL510 but not in other genotypes. Cluster-1 is highlighted by known indicators of oxidative stress such as (-)-allantoin (Kand'ár and Žáková, 2008) and distinguishes the super-sensitive FL499 from the others. Cluster-3 distinguished the tolerant FL478 from

316 the rest, enriched with JA-associated metabolites and fatty acids.

ABA and other related metabolites are the dominant components of cluster-4. This cluster is most similar between the tolerant parent Pokkali, sensitive parent IR29

319 and tolerant FL478, and separated the super-tolerant FL510 and super-sensitive FL499

320 from the group. Cluster-5 features the compatible osmolyte trehalose and other

321 compounds with similar properties and distinguished the sensitive parent IR29. Overall, 322 metabolite profiles revealed meaningful enrichments that distinguished the 323 transgressive segregants. PCA indicates that the salt-sensitive parent IR29 has 

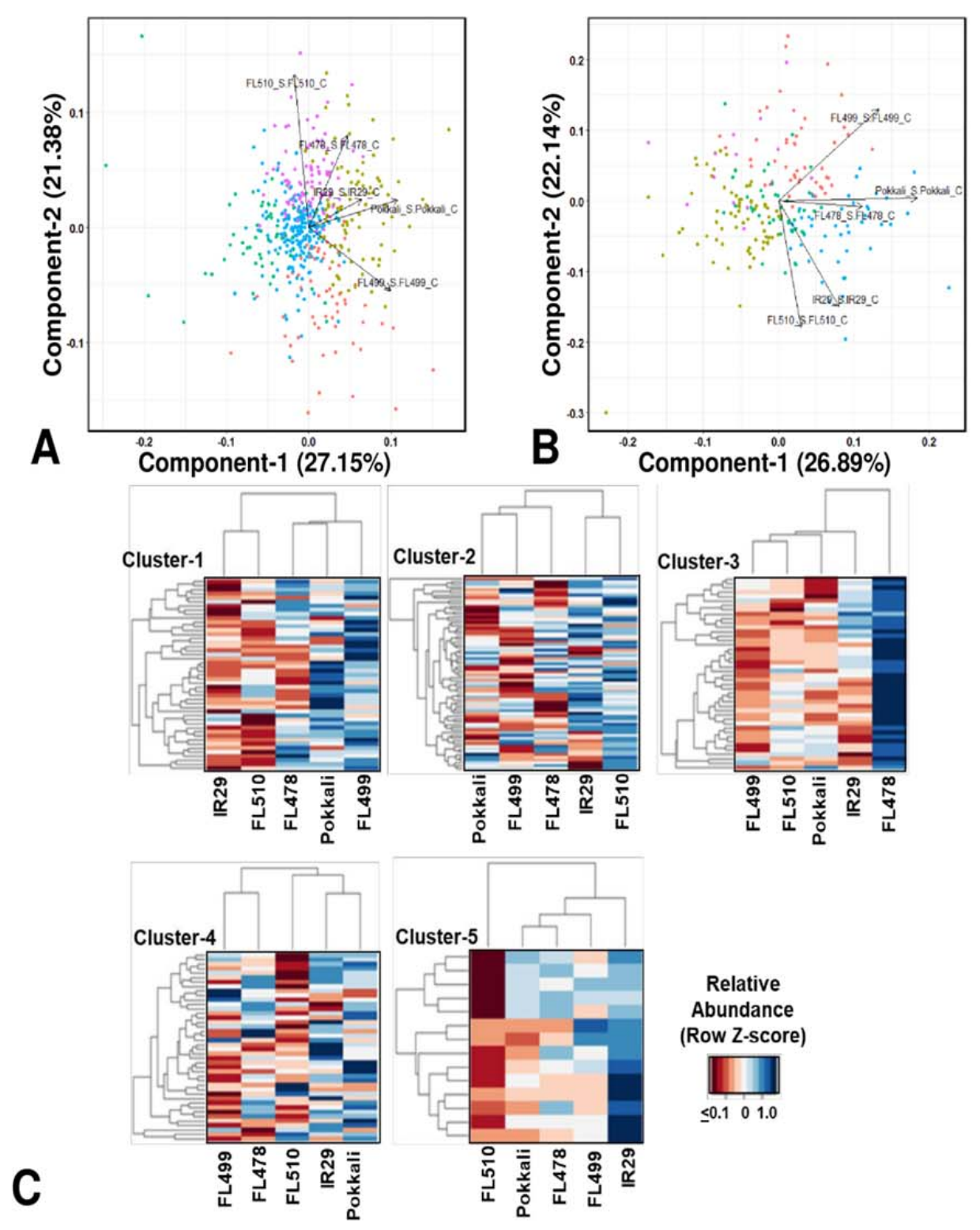

Figure 4. Shotgun LC-MS/MS metabolite profiles across the representative phenotypic classes at the point of maximum stress (7 days or $144 \mathrm{hr}$ ) at EC= $12 \mathrm{dS} / \mathrm{m}$. (A) Principal component analysis (PCA) of metabolites with common occurrence regardless between control and stress in sensitive parent IR29, tolerant parent Pokkali, sensitive FL454, tolerant FL478, super-sensitive FL499, and super-tolerant FL510. PC-1 and PC-2 separated the good RILs FL478 and FL510 from the poorest RIL FL499, accounting for $27.15 \%$ and $21.38 \%$ of phenotypic variance, respectively. The PCA also shows high similarities between the two parents. (B) PCA of metabolites with differential abundances between control and stress at $p<0.05$. Based on PC-1 and PC-2, which accounted for $26.89 \%$ and $22.14 \%$ of phenotypic variance, respectively, the super-sensitive FL499 was quite distant from the super-tolerant FL510, despite the high level of similarities between the parents, hence non-additive and transgressive. The tolerant FL478 was more similar to Pokkali than to IR29, and any of its siblings. (C) K-means clustering heat maps and dendrograms showing the patterns of metabolite co-abundances across genotypes. Heatmap highlights a cluster that is highly abundant in each genotype. Clustering by genotype shows the similarity of IR29 and FL510, especially in clusters 1,2 , and 4 . Cluster 5 , highlights the differences that are contributory towards contrasting phenotypes. 
bioRxiv preprint doi: https://doi.org/10.1101/2020.06.25.171603; this version posted June 26, 2020. The copyright holder for this preprint (which was not certified by peer review) is the author/funder. All rights reserved. No reuse allowed without permission.

325 with the trends revealed from the real-time growth profiling.

326 
Transcriptome signatures across the gradient of salt tolerance

The leaf transcriptomes of each genotype were profiled in a time-course $(0,24$, 48, 72,144 hr) RNA-Seq experiment to further substantiate the properties of the outliers. The RNA-Seq mapping statistics of 44,199 transcript variants from 14,696 expressed loci are summarized in Supplemental Table 2. Contrasting profiles were evident between IR29 and Pokkali, while similarities and differences were evident across RILs (Figure 5). The super-tolerant FL510 had the highest number of genes with no significant change in expression at EC $=12 \mathrm{dS} / \mathrm{m}$. Upregulation in FL510 occurred gradually but progressively compared to others. In the super-sensitive FL499, only subtle changes occurred during the early stages of stress, but many genes were drastically upregulated or downregulated at 144hr/7-day, coinciding with severe leaf senescence and necrosis. The tolerant FL478 and sensitive FL454 had large number of early, late, sustained, and transiently upregulated and downregulated genes similar to IR29 and Pokkali.

The most significant changes in expression involved genes with important roles

342 in integrating stress and growth responses, including calmodulin-like OsCML27

343 (Os03g0331700) that functions as a sensor for $\mathrm{Ca}^{2+}$-mediated stress signaling

344 (Perochon et al., 2011), high-affinity $\mathrm{K}^{+}$transporter OsHKT7 (Os04g0607600), which is a strongly selective transporter of $\mathrm{Na}^{+}$against $\mathrm{K}^{+}$(Suzuki et al., 2016; Oda et al., 2018), metallothionein OsMTI4A (Os12g0570700) involved in oxidative defenses (Zhou et al., 2006; Yang et al., 2009; Kumar et al., 2012), and the Multi-Pass MYB-transcription factor OsMPS (Os02g0618400) involved in growth regulation (Schmidt et al., 2013). These genes were used to bait for other co-expressed genes (network cohorts) to understand the significance of co-expression signatures across the comparative panel.

The OsCML27 profile was most distinct in the super-tolerant FL510 where it was upregulated in sustained manner (Figure 6A). The parents IR29 and Pokkali did not

353 share any cohort genes with any progeny, while only minimal overlaps at best occurred between FL510, FL478, FL499, and FL454 (Figure 6A). These trends illustrate the nonadditivity (network rewiring) of parental gene expression in the progenies, where outlier

356 trends were evident. The OsCML27 cohorts in FL510 were enriched with salicylic acid

357 (SA) and jasmonic acid (JA) associated genes with sustained upregulation through 144 


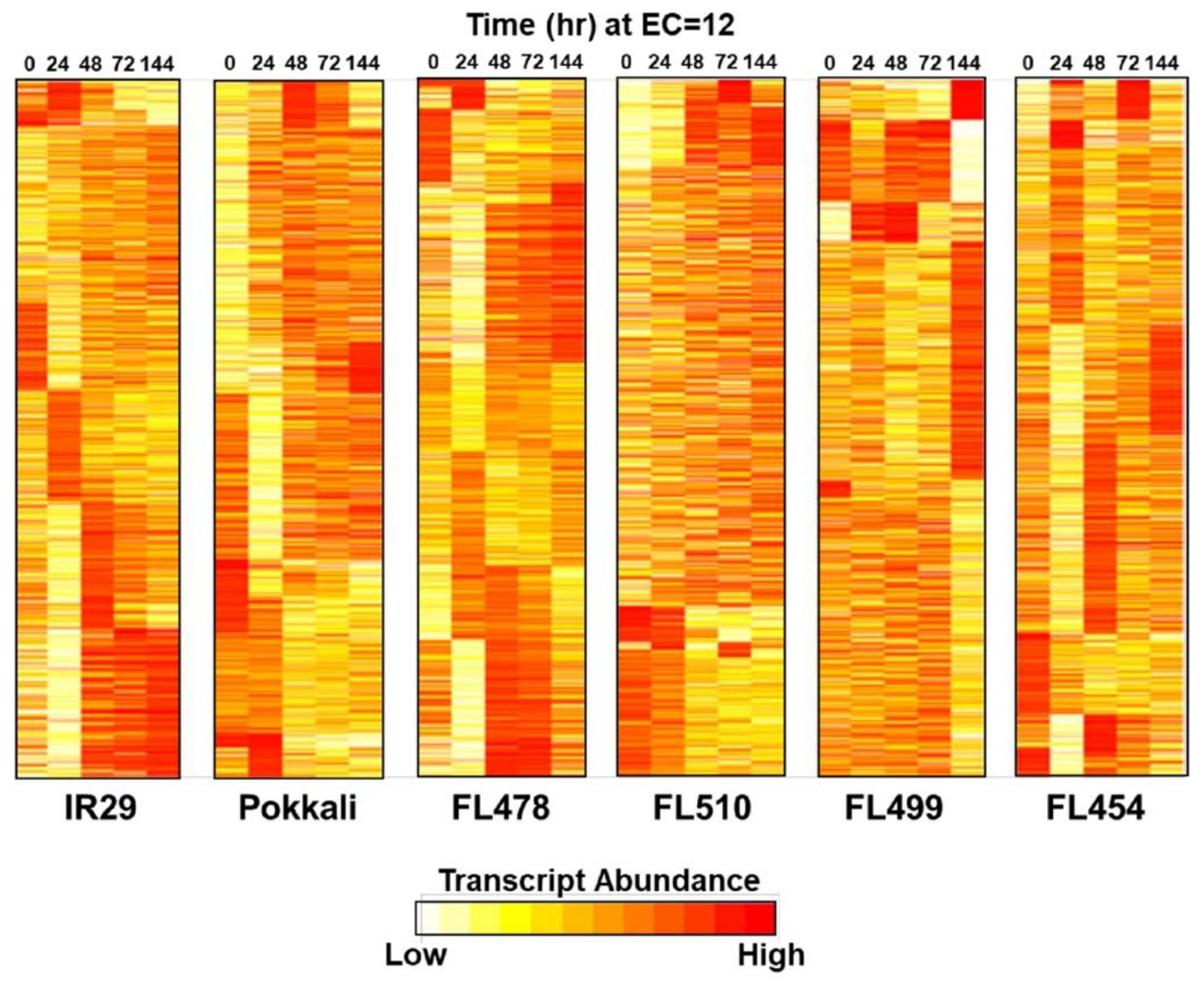

Figure 5. Temporal profiles of salt stress $(E C=12 \mathrm{dS} / \mathrm{m})$ transcriptomes in parents (IR29 = sensitive; Pokkali = tolerant) and their RILs (FL454 = sensitive; FL499 = super-sensitive; FL478 = tolerant; FL510 = super-tolerant). Parallel comparison of transcriptomes across genotype shown as K-means++ clusters included 14,696 unique transcript loci. The super-tolerant FL510 is the most unique, having gradual changes in expression. The other genotypes have large clusters of co-expressed genes with drastic upregulation or downregulation across time.

358 hr (Supplemental Data Set 3). In contrast, distinct subsets of transiently upregulated 359 genes comprised the networks in other RILs, enriched with functions associated with 360 signal transduction, cell wall biogenesis, and growth. Transient expression among the 361 poor RILs suggests a potential interruption of growth under severe stress. In the other 362 poor RILs, the OsCML27 cohorts also included downregulated genes associated with 363 stress adaptation such as OsFBK12 (Os03t0171600-02; Delayed senescence Kelch364 repeat F-box protein), OSISAP1 (Os09t0486500-02; Cold, drought and salt tolerance- 

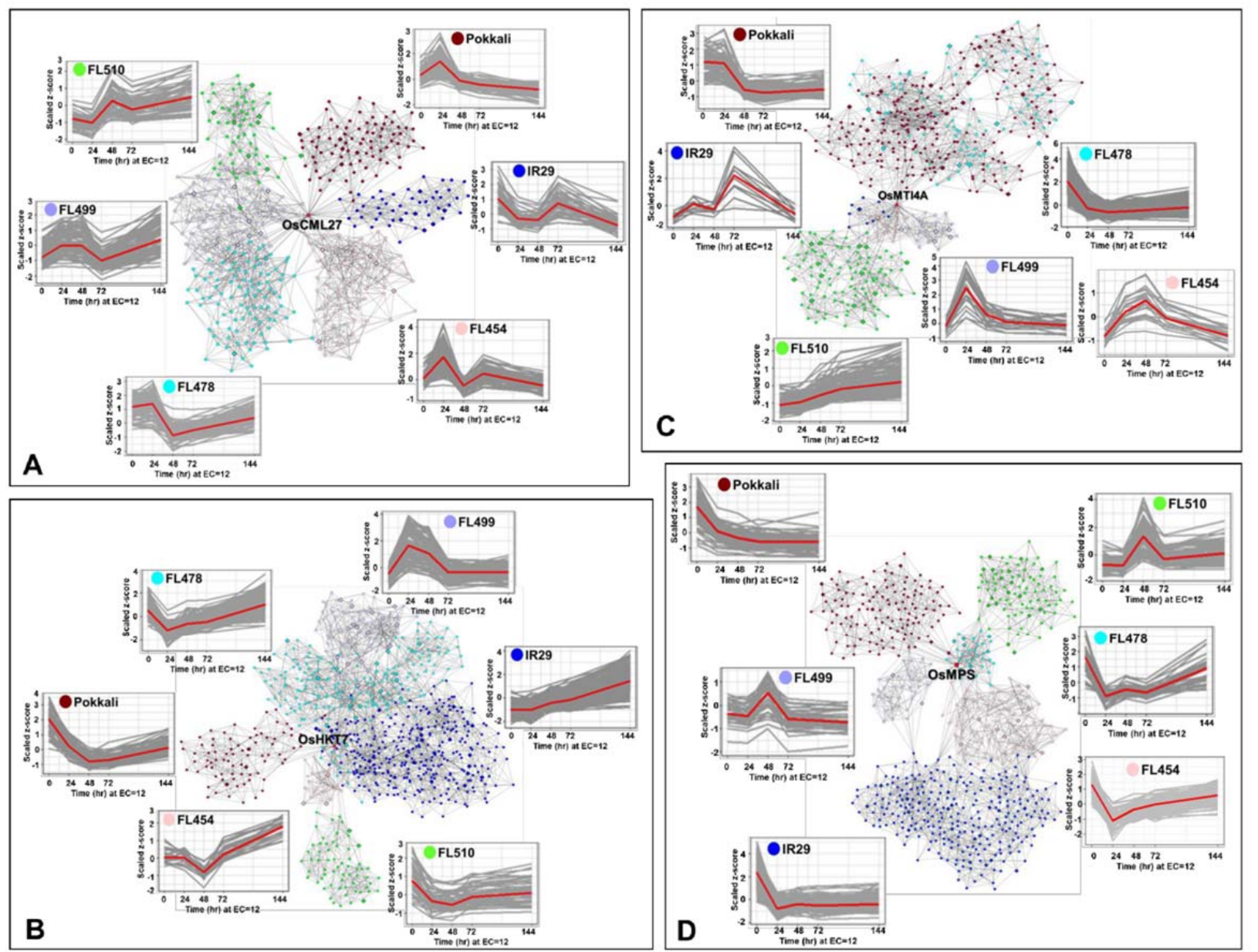

Figure 6. Comparison of transcriptional co-expression networks across parents and their RILs (FL454 = sensitive; FL499 = super-sensitive; FL478 = tolerant; FL510 = super-tolerant). Models represent the networks for: (A) OsCML27, (B) OsHKT7, (C) OsMTI4A, and (D) OsMPS, with important roles in developmental and stress-related responses. The temporal co-expression plots of the bait or core genes (red line) with their cohort genes (gray lines) are given for each network. Connectivity of cohort genes was based on Pearson Correlation Coefficients by mutual rank. Cohort genes are represented by nodes and coexpression is reflected on the edges.

associated A20/AN1 zinc-finger), OsPLC4 (Os05t0127200-02; Salt and dehydration regulated Phospholipase C4), OsNAGK1 (Os04t0550500-05; Drought-regulated Nacetylglutamate kinase-1), OsMST6 (Os07t0559700-00; Monosaccharide transporter involved in water retention and membrane stability), and OsHrd3 (Os03t0259300-01;

369 Unfolded protein and apoptosis response; Supplemental Data Set 3) (Mukhopadhyay et al., 2004; Chen et al., 2013; Ohta and Takaiwa, 2015; Deng et al., 2019; Liu et al.,

371 2019a). The superiority of the FL510 OsCML27 network is further illustrated by the 
372 distinct upregulation of OsIAA1 (Os01t0178500-02; auxin repressor AUX/IAA1),

373 suggesting the interruption of auxin-mediated growth signaling in the poor RILs (Song et 374 al., 2009).

375 The OsHKT7 was upregulated in IR29 but downregulated in Pokkali (Figure 6B).

376 The super-tolerant FL510 resembled the Pokkali profile, while the tolerant FL478 and 377 sensitive FL454 were more similar to IR29 and the super-sensitive FL499 had non378 parental profile. The OsHKT7 cohorts had the most overlap in FL510 and Pokkali, 379 enriched with functions associated with transcription, translation, and cell division 380 (Supplemental Data Set 4). However, the cohorts in FL510 appeared to be uniquely 381 enriched with cation transport functions such as OsMTP9 (Os01t0130000-01) for 382 manganese transport, and OsCDT2 (Os06t0143100-01) for cadmium exclusion 383 (Kuramata et al., 2008; Ueno et al., 2015). The cohorts in IR29, FL454, FL499 and 384 FL478 were associated with stress sensitivity highlighted by OsMYB30 (Os02t0624300385 01) and OSHSFB2B (Os08t0546800-01) (Xiang et al., 2013; Lv et al., 2017).

The cysteine-rich metallothionein gene OsMTI4A was downregulated in Pokkali 387 and transiently upregulated in IR29 (Figure 6C). Expression in FL478 was more similar 388 to Pokkali, while expression in FL499 and FL454 were more similar to IR29. Most 389 interestingly, expression in FL510 was distinct. While significant network overlaps are 390 apparent between FL478 and Pokkali, and between IR29, FL454, and FL499, the cohort 391 genes in FL510 were also distinct from the rest (Supplemental Data Set 5). The Pokkali 392 and FL478 networks included downregulated genes involved in translation, cell division 393 and growth regulation, such as CyclinF2-2 (Os02t0605000-01) and CyclinB1-5 394 (Os05t0493500-00) (La et al., 2006). The networks in the poor genotypes IR29, FL454, 395 and FL499 were comprised of transiently upregulated genes involved in general stress 396 response. The unique OsMTI4A network in super-tolerant FL510 was characterized by 397 sustained co-upregulation of genes involved in hormonal, growth and stress signaling 398 such as BZR3 (Os06t0552300-01; repressor of brassinosteroid signaling), OsIAA13 399 (Os03t0742900-01; Auxin repressor AUX/IAA13), CDKG2 (Os04t0488000-02; cyclin400 dependent kinase G-2), OsE2F1 (Os02t0537500-01; Mitotic cycle E2F protein), and 401 extensin (Os01t0644600-00) (Kosugi and Ohashi, 2002; Tank and Thaker, 2011; Kitomi 402 et al., 2012; Schmitz et al., 2013; Draeger et al., 2015). The novelty of FL510 OsMTI4A 
403

network provides another layer of support to its unique ability to efficiently integrate stress and growth responses.

The MYB-type Multi-pass transcription factor OsMPS is a critical regulator of plant growth and development under stress (Schmidt et al., 2013). While this gene was significantly downregulated in both parents, different patterns of transgressive upregulation were evident across the RILs (Figure 6D). Distinct expression in supertolerant FL510 was characterized by early induction at $48 \mathrm{hr}$. While the OsMPS network appeared to be distinct in each genotype, the most significant overlaps were between the super-tolerant FL510 and tolerant FL478 (Figure 6D). Cohorts in FL510 is enriched for JA and SA signaling genes such as OsWRKY3 (Os03t0758000-01), OsWRKY67 (Os05t0183100-01), OsWRKY53 (Os09t0334500-01), OsWRKY74 (Os05t0343400-01), MYC2 (Os10t0575000-01) and RERJ1 (Os04t0301500-01; Supplemental Data Set 6), which are important for integrating stress with development, and shared at least partially with FL478 (Ramamoorthy et al., 2008; Miyamoto et al., 2013; Ogawa et al., 2017).

The OsMPS network in Pokkali was enriched with genes associated with general transcription and translation, reinforcing the trends observed in its other networks. The other genotypes show few similarities with OsCML27, OsHKT7, and OsMTI4A networks with the downregulated oxidative stress genes such as Prx19 (Os01t0787000-01; peroxidase), Prx117 (Os08t0113000-01; peroxidase), and OsSIK1/OsER2 (Os06t0130100-01; LRR receptor-like serine/threonine kinase), and other genes involved in cell wall biogenesis (Cosio and Dunand, 2008; Ouyang et al., 2010). These trends are consistent with the relatively high growth penalties as shown by real-time growth profiling.

Despite the similar OsMPS expression in the super-sensitive FL499 and supertolerant FL510, much of the cohort genes in their respective networks were distinct. The targets of OSMPS that were co-upregulated in FL510 were not upregulated in FL499. This suggests a possible complete uncoupling of the upstream regulator and its target effectors in FL499, and such uncoupling may only be partial in the other genotypes. Overall, the transcriptional networks support the inherent uniqueness of FL510, due to the combined effects of parentally derived profiles and as well as those that were rewired. 
The correlation coefficients across each network based on median expression values of cohort genes were used as a measure of similarity across the genotypes to further illustrate the transgressive nature of FL510 and FL499 (Figure 7A). FL510 had the lowest correlation with all other genotypes. Similarly, the transgressive nature of the super-tolerant FL510 and super-sensitive FL499 was supported by the correlation coefficients based on the number of cohort genes that overlap across the genotypes, where FL510 and FL499 tend to cluster together (Figure 7B). On the other hand, IR29 and FL478 shared many more genes amongst them than with the other genotypes. FL478 was selected for Saltol and for its overall similarity with IR29.

\section{Transcriptome profiles as window to metabolic status}

Adjustment of growth potential appeared to be critical for the superior salt tolerance of FL510. Such enhanced potential is due to the coupling of good properties

447 from either parent or uncoupling of bad properties from the good properties from the 448 same parent. To address this hypothesis, we assessed the similarities in primary metabolic status by reconstructing models of transcriptional status of glycolysis and tricarboxylic acid (TCA) cycles, starch metabolism, and nitrogen assimilation and transport pathways to gauge the state of plant growth. Pathway status models were based on maximum $\log _{2}$-fold change for the critical genes in each pathway.

In general, glycolysis, TCA cycle, and starch and sugar metabolism were 454 moderately repressed in the tolerant parent Pokkali and super-tolerant FL510, in stark 455 contrast to the inferior genotypes, especially the sensitive parent IR29 and supersensitive FL499 where significant upregulation was quite evident (Figure 8A-C). These

457 trends suggest that metabolic rates are faster in the poor genotypes, perhaps as 458 consequence of the need to rapidly replenish metabolic intermediates as they are constantly depleted by cellular adjustments and defense. Superior genotypes appeared

460 to maintain better balance and less perturbation. Nitrogen metabolic and transport 461 pathways appeared to be enhanced in superior genotypes (Figure 8D). As nitrogen 462 assimilation is critical for vegetative growth, these trends are consistent with the ability 463 to maintain growth even under severe stress, as also revealed by the real-time growth 464 profiling. 

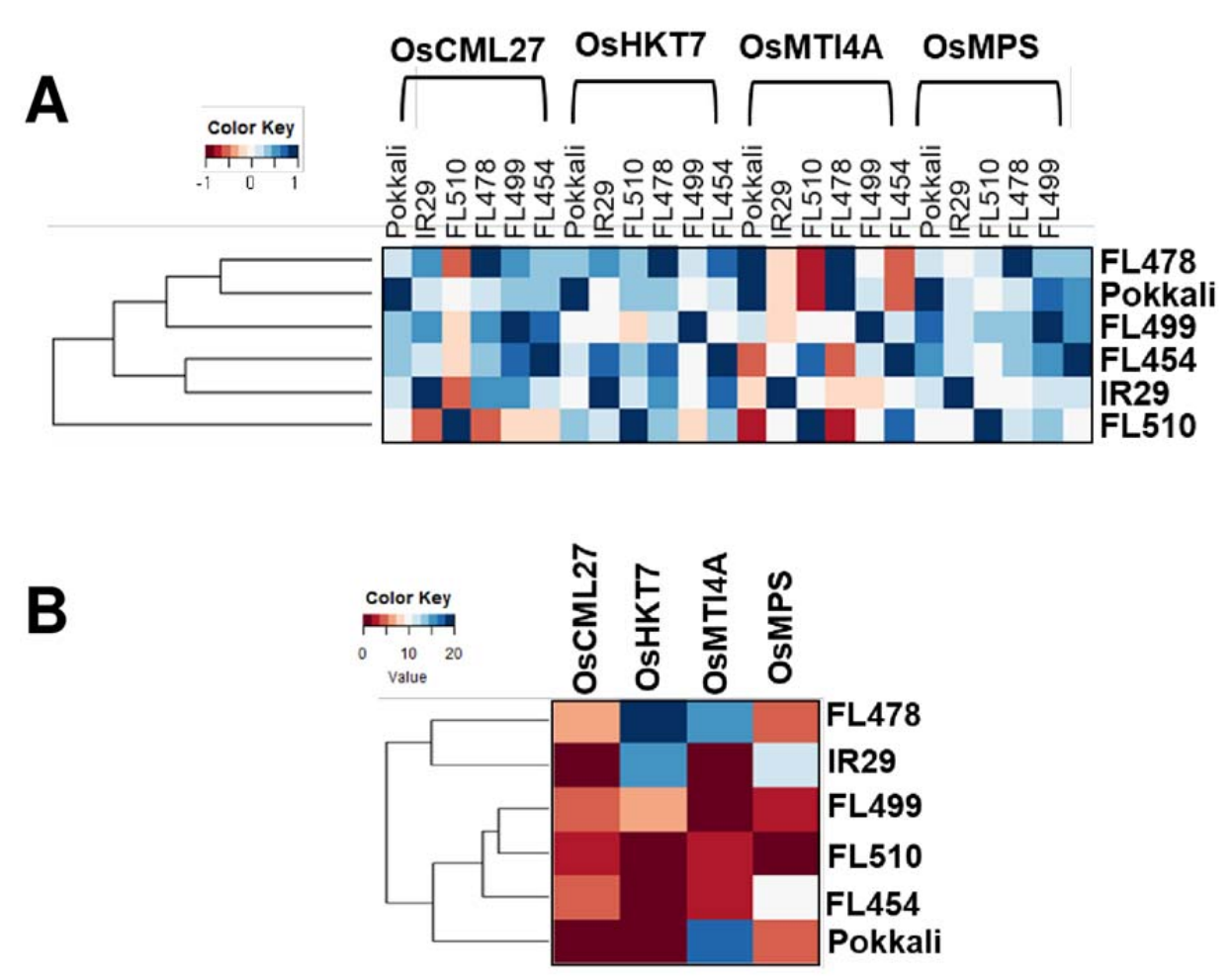

Figure 7. Relatedness of the genotypes according to the gene networks in Figure 6. Similarities among the genotypes were assessed by coefficient of correlation among median expression values of network components (A), and by the number of shared cohort genes (B).

In terms of the primary metabolic profiles, the super-tolerant FL510 was more

466 similar to the tolerant parent Pokkali (Figure 8A-D). This trend only deviated for the

467 Calvin cycle, where FL510 was more similar to the sensitive FL454 (Supplemental

468 Figure 3). However, FL454 and FL510 clustered together with Pokkali, and which still

469 points to a general slow-down or modulation of the Calvin cycle compared to other

470 genotypes. Overall, modulation of metabolic pace in FL510 may be advantageous to

471 maintain homeostasis under conditions when perturbed physiological state.

472

473 


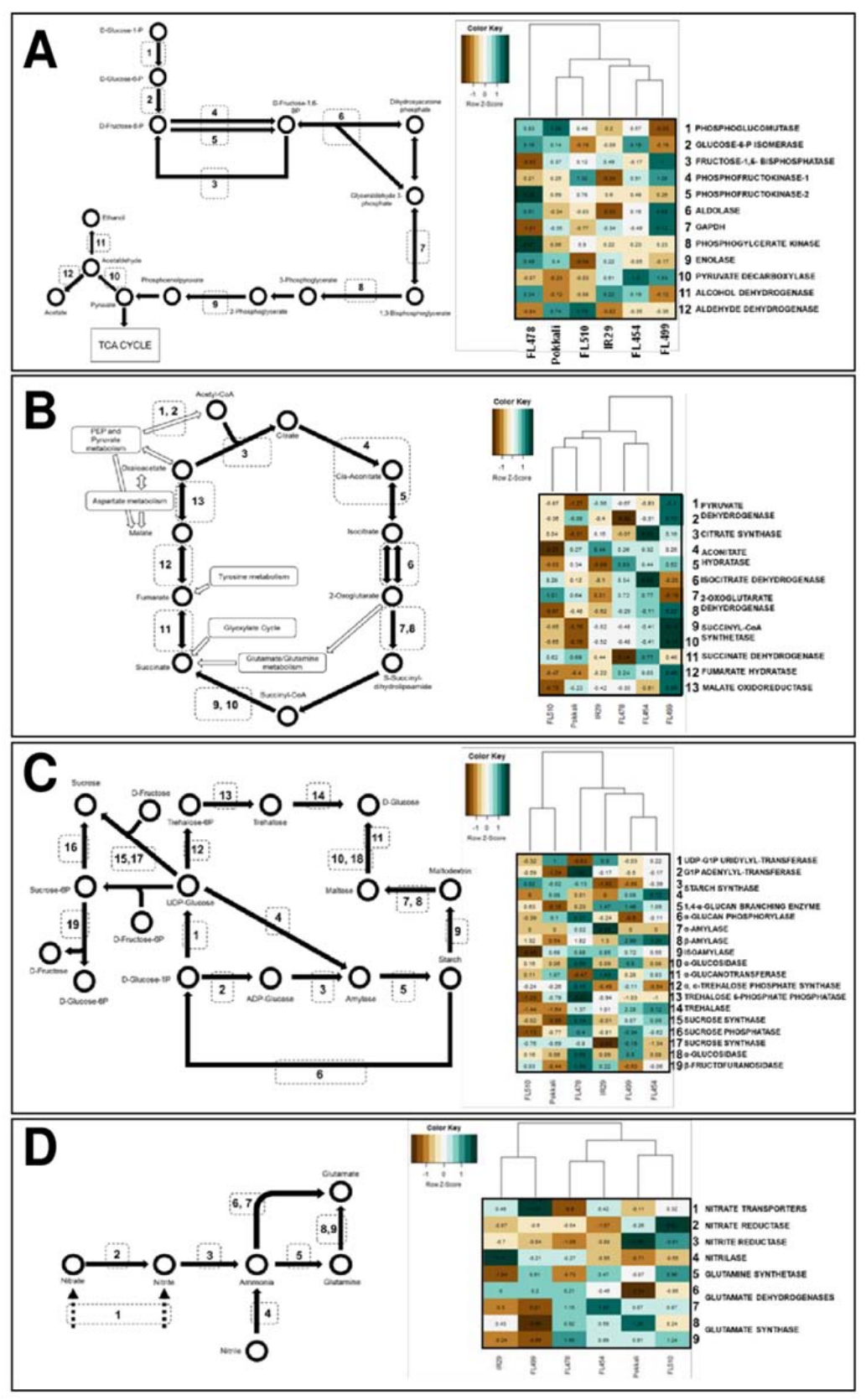

Figure 8. Metabolic similarities based on transcriptome profiles. KEGG models of metabolic pathways are shown on the left panel for glycolysis (A), TCA cycle (B), starch/sucrose metabolism (C), and nitrogen assimilation (D). Transcripts for enzymes in each step were mapped against each pathway. Pathway activities and the pattern of similarities across genotypes are depicted by hierarchical clustering dendrograms based on fold-change in transcript abundances at the time-point with the widest difference from control. FL510 and Pokkali share similarities in repression of the carbon metabolism pathways, while nitrogen assimilation is induced in both genotypes. In comparison, the poor genotypes cluster together.

\section{Discussion}


Evolutionary biology referred to the outlier phenotypes in natural populations as genetic novelties (Wagner and Lynch, 2010). Transgressive segregation is a major source of such genetic novelties, providing the raw materials for adaptive speciation under ecological niches where the majority is less adapted (Rieseberg et al., 1999). The genetic basis of transgressive segregation has been attributed to complementation and additive effects, and positive or negative epistatic interactions, among other phenomena (Dittrich-Reed and Fitzpatrick, 2013). In plant breeding, where directed mating of two individual genotypes (intra-species or inter-species) with wide phenotypic contrasts serve as foundation for stacking desired traits together, transgressive segregation could be a common occurrence. However, much of these novelties are often undetected due to small population size, narrow scope and limited resolution of phenotypic classification, limited generation time to break genetic linkage and physiological drags, and lack of sufficient data and genetic models for their prediction. Nevertheless, when interfaced with the high-resolution genetic scrutiny through genomic modeling, transgressive segregation could provide a fine-tuned combination of compatible and additive traits to address the needs for enhanced environmental resiliency of $21^{\text {st }}$ century crops, above and beyond what could realistically be achieved by transgenics and gene editing (de los Reyes, 2019).

In marker-assisted plant breeding, crosses between parents carrying few

494 complementary major-effect QTL are often made to maximize genetic gains from the 495 combining potentials of the parents, especially in the absence of linkage drags (Collard and Mackill, 2008). While much success has been achieved particularly for adaptive

497 traits and yield potential using this paradigm, the strategy of QTL stacking is often

498 performed with the simplistic expectations that most outcomes are positive additive 499 effects, while ignoring much of the potential negative interactions that undermine the full

500 force or potential of positive synergies. Negative interactions are in fact coming from the 501 background (minor effect QTL or peripheral components) that cannot be explained by 502 the major-effect QTL (i.e., Omnigenic Theory).

503 Focused on a well characterized genetic population of rice (IR29 x Pokkali RILs), 504 this study was motivated by the recognition that the maximum combining potentials of 
equal contribution of the positive additive components (major and minor effects) from either parent, and the absence of antagonistic elements from both parents that undermine the full force of positive synergies. This study also aimed to uncover evidence of genetic network rewiring based on non-parental physiological, biochemical, and molecular attributes. The ultimate goal was to illuminate the various types of synergies that create either large positive or negative net gains.

We addressed the physiological coupling and uncoupling hypothesis by revealing

513 that IR29 (sensitive parent) was the source of positive growth and developmental

514 attributes that were complemented by the superior defense attributes of Pokkali

515 (tolerant parent). Additive effects are manifested because of the absence of

516 physiological drags that undermine the full expression of growth and developmental

517 attributes in IR29, and the defense attributes in Pokkali either independently or through

518 their interactions. Evidences of non-parental physiological, biochemical, and molecular

519 attributes were uncovered in the transgressive segregants, consistent with the proposed

520 network rewiring theory (de los Reyes, 2019).

$521 \quad$ To understand the subtleties of physiological coupling and uncoupling, we

522 borrowed the paradigm of 'personalized genomics medicine', which considers any two

523 individuals with similar phenotypes to have unique signatures based on multi-

524 dimensional or holistic criteria. We scrutinize the representatives of the full phenotypic

525 range across a recombinant inbred population at the highest resolution, which is not

526 normally achieved in conventional plant breeding pipeline. Our results further

527 illuminated the biological basis of transgressive segregation to complement what is

528 known in classical plant breeding. This study provides a solid proof of concept that

529 genetic novelties can be created by hybridization. It represents an important advance

530 for further exploration of transgressive segregation towards novel adaptive phenotypes

531 that may not be achieved by functional genomics, transgenics or gene editing.

533 Coupling and uncoupling effects at multiple levels

$534 \quad$ The transgressive properties of the super-tolerant FL510 stems mainly from the 535 complementation of beneficial traits (coupling) and shedding of potential drags 536 (uncoupling) from both parents, made possible by the fortuitous recombination events 


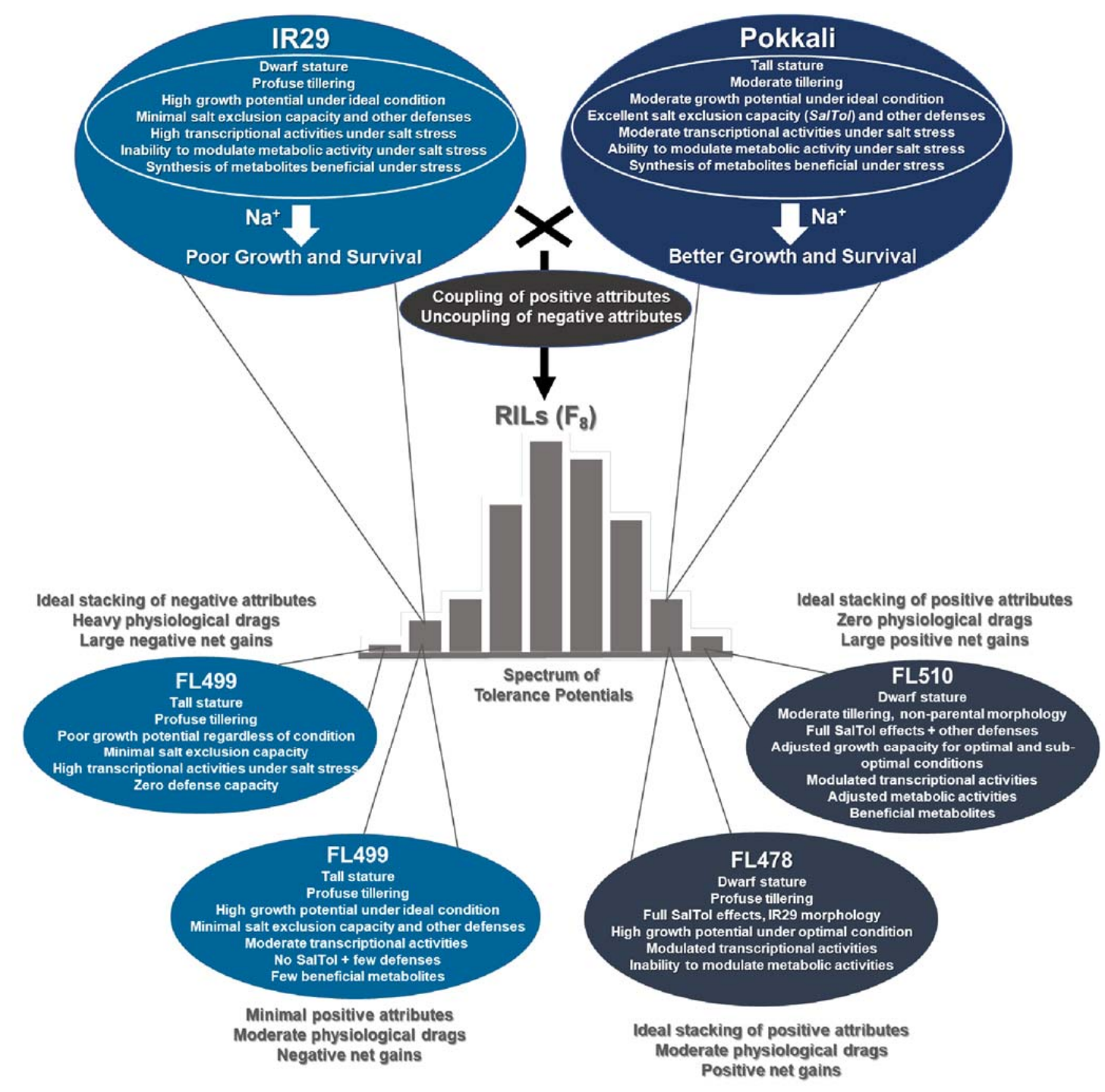

Figure 9. Hypothetical model of physiological coupling and uncoupling in transgressive segregants for salt tolerance across the IR29 x Pokkali recombinant inbred population based on macrophysiological, biochemical and molecular profiles according to De los Reyes (2019). This model proposes that the novelties of FL510 and FL499 are due to the coupling in the progeny of the good properties coming from either parent or uncoupling of bad properties from the good properties from the same parent. On top of the core mechanisms that contribute to a large proportion of phenotypic variance for defense potentials, each parent has their own characteristics that may or may not be beneficial under stress. Benefit from IR29 would be its superior growth and development potentials. Pokkali offers many stress defense mechanisms including salt exclusion. Combining the physiological potentials of parents with the reconfigured (non-parental) properties led to positive or negative coupling and uncoupling effects in RILs.

537 and genome shuffling through at least eight generations $\left(F_{8}\right)$. Coupling and uncoupling

538 were observed at multiple levels, i.e., macro-physiological, metabolome, and

539 transcriptome. A hypothetical model is presented in Figure 9 to illustrate how the unique 
assemblages of properties or their opposites from each parent configured unique attributes to certain individuals.

At the macro-physiological level, the best measure of variation for salt tolerance was the magnitude of growth penalty. Sub-optimal conditions slow-down the rate of

544 photosynthesis, while requiring more resources to feed into the maintenance of homeostasis and short-term defenses (Munns and Gilliham, 2015). High-resolution

546 phenotyping indicates similarity in the stress response capacity of FL510 and Pokkali,

547 but a clear difference in overall performance. These genotypes had the highest

548 aggregate phenotypic scores (APS), indicating that FL510 inherited the stress-related

549 traits from Pokkali, most prominently the salt exclusion capacity through Saltol (S1A-B

550 Figure). Evidently, the tolerant genotypes (Pokkali, FL510, FL478) clustered together

551 when only salt exclusion is considered (Figure 2). FL478 has the Saltol effects yet

552 outperformed by FL510, indicating that factors above and beyond salt exclusion

553 maximize tolerance potential.

It is important to note that there appears to be a threshold as to how much the salt exclusion alone can effectively provide protection if other positively affecting and non-antagonistic properties are not in place. Prolonged exposure to stress will eventually damage the plant regardless of its inherent defense potential through salt exclusion. One example related to the damage incurred eventually relates to the reduction of transpiration, as water and nutrient uptake together with gas exchange still needs to happen for maintenance. Thus, salt is still inevitably transported into the plant along with its toxic effects.

Real-time growth profiling indicated that the morpho-developmental attributes inherited by FL510 from the salt-sensitive parent IR29 are positively complementing other attributes from the salt-tolerant parent Pokkali, i.e., synergism. For instance,

565 smaller stature and low tillering habit distinguish FL510 and FL478 from their inferior 566 siblings, and these traits appeared to offer fitness benefits as they are much easier to 567 support energetically under stress when metabolic intermediates are constantly being 568 diverted towards cellular adjustments and defenses. In contrast, the inferior genotypes 569 FL454 and FL499 appeared to exhibit less capacity for growth modulation, as indicated 570 by tall stature (Pokkali), profuse tillering habit (IR29), or both (FL454, FL499). Survival is 
571 prioritized over continued growth under stress; hence the growth curve plateaus

572 prematurely. Higher PSA ratio was observed in IR29, Pokkali, and FL478 during the

573 onset of stress compared to FL510, FL454, and FL499, possibly reflecting an attempt to

574 increase water uptake and turgor pressure for short-term mitigation of osmotic stress.

575 The deviation ('forking') of growth curves between stress and control is a measure of how much metabolism has been hampered, and the penalty incurred due to

577 perturbed cellular processes and injury. Being the major trade-off of survival, this 578 deviation was minimal in tolerant genotypes especially FL510. In this context, the coupling of the morpho-developmental features of IR29 with the stress-response

580 mechanisms of Pokkali is ideal, allowing the plant to maximize its survival responses, while minimizing the growth trade-offs by reaching the peak of vegetative growth earlier with less metabolic costs. The super-sensitive FL499 needs to cope with stress through an inferior mechanism inherited from IR29, while trying to maintain its inherent high growth capacity. This leads to a drastic decline in growth rate and extreme sensitivity to salt.

Metabolite profiles also support the idea that IR29 contributes complementary

587 factors towards a transgressive phenotype. Metabolites were similarly expressed

588 between FL510 and IR29, indicating beneficial factors from IR29. Meanwhile, cluster-5

589 indicates the 'drags' that have been uncoupled, further contributing to increased positive

590 net gain in FL510. Cluster-4 could also represent some of the 'drags' that prevented

591 FL478 from achieving its full potential. These results further support the hypothesis that

592 coupled beneficial factors from both parents are essential in creating an ideal synergy

593 and maximal net gain.

$594 \quad$ The transgressive nature of the super-tolerant FL510 is further highlighted by its

595 unique transcriptome signatures. The robust response in FL510 represents an optimal

596 mechanism characterized by steady and progressive upregulation or downregulation

597 across time. Responses are either for long-term adaptation or short-term adjustment (de

598 los Reyes et al., 2018). Gene expression in the other inferior genotypes occurred in less

599 orderly fashion compared to the gradual but progressive nature in FL510, indicative of

600 perturbed state rather than adaptation. 
Comparison of transcriptional networks for four genes (OsCML27, OsHKT7, OsMTI4A, OsMPS) with important roles in development and stress also highlight the unique configuration of FL510 as the most deviant to the parental profiles, hence rewired regulatory network as an outcome of recombination. FL510 also inherited the propensity for repressed carbon metabolism and enhanced nitrogen metabolism of Pokkali. The attributes inherited from both parents led to improved potential in combination with reconfigured gene networks gained through rewiring. All these factors contributed to synergies that allow robust growth of FL510 even under salt stress, through efficient integration of developmental and stress-related responses (de los Reyes et al., 2018). In contrast, the inferior siblings have either incomplete inheritance of beneficial characters (e.g., FL478), stacking of detrimental characters that maintain the sensitivity properties of IR29 (e.g., FL454), or further stacking of physiological drags

613 that exacerbate the baseline sensitivity inherited from IR29 (e.g., FL499). In the case of 614 FL499, profuse growth was unsustainable, aggravated by the inability to adequately address the toxic effects of $\mathrm{Na}^{+}$.

As bottom line, the super-tolerance of FL510 and super-sensitivity of FL499 can be defined by multiple coupling and uncoupling of physiological and molecular mechanisms, hence net gains (Figure 9). In FL510, the synergism of parental attributes was evident. Most apparent is the synergy of morpho-developmental features from IR29, and salt exclusion mechanism from Pokkali. While FL478 had similar traits as FL510, it is slightly polluted with residual physiological drags that lower its overall potential, as indicated by the metabolite and transcriptome data. FL478 also had the

623 morpho-developmental attributes of IR29 (e.g., tillering capacity). Evidently, sensitive 624 genotypes are compromised by an unsustainable metabolic requirement owing to their very active growth, while lacking the effective stress response mechanisms of Pokkali.

At the transcriptome level, the ideal synergism in FL510 allows for a sustained response through the stress period. In contrast, inferior genotypes must respond immediately, leading to an overreaction, which is energetically wasteful and detrimental

629 in the long-term. If the stress is not relieved, the net result is a deficit that may not be 630 reconciled, thus large penalties to survival and growth. The chances that extreme phenotypes such as those in FL510 and FL499 would occur in a population are small, 
bioRxiv preprint doi: https://doi.org/10.1101/2020.06.25.171603; this version posted June 26, 2020. The copyright holder for this preprint (which was not certified by peer review) is the author/funder. All rights reserved. No reuse allowed without permission.

632 as there needs to be a specific combination of parental traits and creation of novel 633 regulatory networks to create an ideal or non-ideal synergy. 
Relevance of the coupling and uncoupling effects to the Omnigenic Theory

The Omnigenic Theory represents a modern paradigm for explaining the genetic causes of complex diseases in humans that cannot be fully explained by polygenic effects (Boyle et al., 2017). According to this theory, complex traits are controlled by thousands of genes or loci scattered throughout the genome, rather than a small group of genes detected by conventional QTL mapping or genome-wide association. Core genes/loci are central to the trait and detected as QTL or major genes by forward genetic screens. The peripheral genes/loci are below the threshold of QTL detection, as they have immeasurable effects as individual components of a network. However, their combined effects can be equal to or more than the effects of the cores (Liu et al., 2019b). To maximize the potential for trait expression through $G \times E$, both the combinations of the core and peripheral alleles and their interactions must be optimal. compatible traits from both parents (Rieseberg et al., 1999). Their novelties are also due to the absence of physiological or genetic linkage drags. However, if fortuitous, uncoupling events could also result in the loss of positive synergies (de los Reyes, 2019). This study illustrated that complementation in either synergistic or antagonistic fashion creates transgressive properties at both sides of the phenotypic spectrum, extending beyond the typical salt exclusion mechanism contributed by Pokkali. In FL510, growth reduction was minimized since metabolic impairment was not as extensive compared to other genotypes. FL478 and Pokkali both possess salt exclusion mechanisms; however, they lack the multitude of complementary but minute effects for

657 further incremental enhancements.

In FL510, the synergism among the positive parental attributes buoy plant health enough to survive under stress. Salt exclusion mechanism reduces $\mathrm{Na}^{+}$toxicity in

660 metabolically active tissues, which works together with the lower metabolic requirement 661 for growth and development, hence a robust response. Transport of $\mathrm{Na}^{+}$and $\mathrm{Cl}^{-}$alone 662 constitutes major cellular energy costs. When minimized, it allows the plant to survive

663 longer under saline environment (Tyerman et al., 2019; Munns et al., 2020). The 664 collective responses uncovered in FL510 are consistent with the Omnigenic Theory, as 665 no single mechanism is adequate to explain its extreme phenotype. The same thing is 
666 true for the super-sensitive FL499 with antagonistic effects. Physiological gain is the

667 result of ideal synergy, reflecting the concept that the majority of the genome

668 contributes to the full physiological potential of each individual in a population.

\section{Comprehensive phenotyping and genomic modeling of transgressive traits}

The transgressive salt tolerance of FL510 may not extend to other types of stress

672 such as drought. Indeed, other RILs derived from IR29 x Pokkali have been shown to 673 survive under severe water-deficit better than FL510 (Gendron, 2019). While cases of 674 transgressive properties such as those observed in FL510 and FL499 may be rare in a

675 population, individuals that exceed the parental range may be more common than

676 perceived. Repeated recombination events can create incremental improvements that

677 allow individuals to have slight advantages over their parents. Often, individuals that

678 display such properties are cast aside, as they do not offer much advantage in a 679 constrained plant breeding pipeline. These individuals also often carry undesirable

680 'linkage drags'. However, these individuals offer invaluable tools in modeling how transgressive phenotypes arise in a population.

Genomic selection promises to identify desirable but rare recombinants for traits governed by complex multi-loci interactions by taking into consideration the core and peripheral components and all forms of interactions (de los Reyes, 2019). Increasing the accuracy of modeling is dependent on the number of individuals in the modeling population. With enough individual data points, a wider coverage of possible permutations of recombinants can be mined for genomic patterns that lead to transgressive phenotypes. Thus, the interaction networks from both core and peripheral components may be modeled for their individual weights.

It must be remembered that the process from which transgressive individuals

691 arise in plant breeding is similar in many ways to the process by which new

692 phylogenetic lineages could develop in natural populations through hybridization

693 (Rieseberg et al., 1999; Dittrich-Reed and Fitzpatrick, 2013). The fact that transgressive 694 individuals can arise through repeated recombination events is a hopeful observation. 695 All progeny in a cross is potentially useful for modeling transgressive phenotypes. Each 696 recombination event adds a possible genomic permutation, and these should not be 
discarded, as various types of synergism can be identified and understood from such individuals.

It is also important to increase the resolution of analysis with multiple layers of data across a population. A multi-tier view of phenotypic range will allow a more comprehensive integration of the 'omics' data to unravel the wirings of transgressive phenotypes, analogous to the paradigm of personalized genomic medicine. This study represents a serious attempt to test this approach that is unconventional even in modern plant breeding pipelines, i.e., analyzing multiple layers of data to decipher complementary or antagonistic effects. While it has limitations, future advances in technology will allow a more seamless data integration. Addition of other layers, such as epigenomic configuration and chromatin structure will certainly enhance the prospect of modeling (de los Reyes, 2019). Additionally, beneficial traits do not come solely from one parent, and the value of a genotype as donor cannot be predicted based on phenotype alone, unless its combining potential has been examined in recombinants.

711 Extensive phenotyping may reveal cryptic characters that are beneficial but are often

712 overshadowed by other negative traits.

Materials and Methods

\section{Phenotypic evaluation of the RIL population}

The RILs of IR29 (Xian/indica; salt-sensitive) x Pokkali (Aus; salt-tolerant)

717 consisted of 123 individuals as the core QTL mapping population. Segregation for salt

718 tolerance was established earlier at IRRI based on Standard Evaluation Score (SES)

719 and shoot $\mathrm{Na}^{+} / \mathrm{K}^{+}$at V1 to V5 for the fine-mapping of Saltol at EC= $9 \mathrm{dS} / \mathrm{m}$ (Singh et al.,

720 2007; Thomson et al., 2010). Based on IRRI's data, a comparative panel of 67

721 individuals representing the full range of SES and $\mathrm{Na}^{+} / \mathrm{K}^{+}$was subjected to a multi-

722 parameter evaluation at V4 to V12. The replicated $(n=8)$ experiments were conducted in

723 hydroponics at $30-35^{\circ} \mathrm{C}$ day, $24-26^{\circ} \mathrm{C}$ night; $20 \%$ to $30 \% \mathrm{RH}$; $12-\mathrm{hr}$ photoperiod with

$724500 \mu \mathrm{mol} \mathrm{m} \mathrm{s}^{-2}$ light intensity. Physiological parameters included SES (Gregorio et al.,

725 1997), $\mathrm{Na}^{+} / \mathrm{K}^{+}$, electrolyte leakage index (ELI) (Ballou et al., 2007; de los Reyes et al.,

726 2013), peroxidase (POX) activity, lipid peroxidation (LP) (Jambunathan, 2010), and

727 shoot biomass. 
Seedlings were grown in standard peat moss for fourteen (14) days. Individual plants were transplanted to 0.6-gallon hydroponic buckets with $1 \mathrm{~g} / \mathrm{L}$ Peter's supplemented with $0.4 \mathrm{~g} / \mathrm{L} \mathrm{FeSO}_{4} \cdot 7 \mathrm{H}_{2} \mathrm{O}$. Plants were subjected to salt stress at tillering

732 stage (V4 to V12; (Counce et al., 2000) with 120mM NaCl (EC= $12 \mathrm{dS} / \mathrm{m})$. Samples for

733 physiological assays, RNA extraction, and biomass measurements were collected at 0

$734 \mathrm{hr}$ (control) and 24, 48, 72, and $144 \mathrm{hr}$ after stress.

Shoot biomass ratio, stomatal conductance, and standard evaluation score (SES) $0 \mathrm{hr}$ and $144 \mathrm{hr}$ after stress. Tissue samples were oven-dried $\left(50^{\circ} \mathrm{C}\right)$ for five days and weighed to calculate the $\%$ biomass. Biomass ratio $>1$ indicates growth, while ratio $<1$ indicates penalty. Stomatal conductance was measured using the SC-1 Leaf Porometer (Meter Group Inc., Pullman, WA). These measurements were taken at $0 \mathrm{hr}$ and $144 \mathrm{hr}$ and presented as control/stress ratio. Stomatal conductance was measured at the same time of the day to prevent diurnal effects. At the end of the stress treatments (17 days),

744 the overall health of each plant was rated visually using IRRI's Standard Evaluation

745 System (SES) (Gregorio et al., 1997). SES is based on a ranking of 1 to 9 with higher score indicating more severe magnitude of stress injury.

\section{Electrolyte leakage index} from osmotic stress as well as ion uptake according to Ballou et al. (2007) and de los Reyes et al. (2013). Briefly, leaf discs were sampled from individual plant replicates $(n=$ 5) and placed in $5 \mathrm{~mL}$ ultrapure water (18 megaOhms). Electrical conductivity (EC) was

753 measured with a conductivity meter (Thermo Scientific, Waltham, MA) after allowing the

754 tissue electrolyte to leach out (stress EL). Total tissue electrolytes were measured after

755 boiling at $95^{\circ} \mathrm{C}$. Electrolyte leakage was calculated as: $E L_{\text {sample }}=E C_{\text {boiled }} / E C_{\text {unboiled }} X$

756 100. ELI was calculated with the following equation and expressed as a mean of ELI (n

$757=5): E L I=E L_{144 h r} / E L_{\text {ohr }} \times 100$. 


\section{Peroxidase activity assay}

Total peroxidase activity (POX) was measured with the Amplex® Red Hydrogen Peroxide/Peroxidase Assay Kit according to manufacturer's instructions (Invitrogen, Carlsbad, CA). Pulverized leaves were homogenized in 20mM sodium phosphate buffer $(\mathrm{pH} 6.5)$ and centrifuged at $9000 \times \mathrm{g}$ for $10 \mathrm{~min}$ at $4^{\circ} \mathrm{C}$. Samples were reacted with the Amplex® Red mix in the dark for $30 \mathrm{~min}$, and absorbance at $560 \mathrm{~nm}$ was presented as means $(n=3)$. POX was based on a standard curve of pure horseradish peroxidase at a range of 0 to $2 \mathrm{mU} / \mathrm{mL}$.

\section{Lipid peroxidation assay}

Assays for lipid peroxidation were adopted from Jambunathan (2010). Leaf samples were pulverized in liquid nitrogen, homogenized with $0.1 \%$ trichloroacetic acid (TCA), and centrifuged at 10,000 x g for $15 \mathrm{~min}$ at room temperature. The supernatant was combined with $20 \%$ TCA and $0.5 \%$ thiobarbituric acid (TBA) and incubated at $95^{\circ} \mathrm{C}$

773 for $30 \mathrm{~min}$. Absorbance was measured at $530 \mathrm{~nm}$ and $600 \mathrm{~nm}$, with $\mathrm{A}_{600}$ serving as a

774 background absorbance against $A_{532}$. Values were used to determine the

775 malondialdehyde (MDA) content as an estimate of broken lipid membranes reacting 776 with TBA. MDA content was based on Beer-Lambert equation: $\boldsymbol{C}=\boldsymbol{A} / \boldsymbol{\varepsilon} \mathbf{\times} \boldsymbol{l}$, where $A=$ 777 differences in absorbance at $530 \mathrm{~nm}$ and $600 \mathrm{~nm}, \varepsilon=$ extinction coefficient of $155 \mathrm{mM}^{-}$ $778{ }^{1} \mathrm{~cm}^{-1}, I=$ length $(\mathrm{cm})$ of light path, and $C=$ content $(\mathrm{mM})$ of MDA.

\section{$\mathrm{Na}^{+}$and $\mathrm{K}^{+}$quantification}

783 P-4-20 (A\&L Plains Analytical Laboratory, Lubbock, TX). Values were presented as \%

$784 \mathrm{Na}^{+}$or $\mathrm{K}^{+}$per gram of sample $(\mathrm{n}=3)$. Total $\mathrm{Na}^{+}$and $\mathrm{K}^{+}$were also determined for image785 based phenotyping experiments. Pulverized tissues were digested with dilute $\mathrm{HNO}_{3}$

$786(0.5 \mathrm{M})$ at room temperature and the supernatant was used for flame photometry using 787 the Model 420 Flame Photometer (Sherwood Scientific Ltd., Cambridge). $\mathrm{Na}^{+}$and $\mathrm{K}^{+}$ 788 concentrations were calculated based on standard solution with $0.5 / 1 \mathrm{mM} \mathrm{NaCl}: \mathrm{KCl}$ 789 using the same sample dilution factor, amount of digestion solution, and initial sample 
790 weight as follows: $\left[\mathrm{Na}^{+}\right] /\left[\mathrm{K}^{+}\right]=$Reading/100 $\times$Standard/100 $\times$Digestion solution $\mathrm{x}$

791 Dilution factor/Dry weight x $100(n=5)$.

Proline content

Total proline content of leaves (V7 to V9) was determined according to Bates et al. (1973). Briefly, pulverized leaf tissues (100 mg) were homogenized in $5 \mathrm{ml}$ sulfosalicylic acid (3\% w/v) and then centrifuged at 13,000 x g for $10 \mathrm{~min}$ to collect the supernatant. The assay solution was comprised of fresh ninhydrin $(2.5 \% \mathrm{w} / \mathrm{v})$, glacial acetic acid (60\% v/v), and phosphoric acid (40\% v/v). Supernatant $(0.1 \mathrm{ml})$ was combined with $0.2 \mathrm{ml}$ assay solution and $0.2 \mathrm{ml}$ glacial acetic acid and was incubated at $95^{\circ} \mathrm{C}$ for an hour. Chromophore was extracted with $1 \mathrm{~mL}$ toluene, and the organic phase $(0.1 \mathrm{ml})$ was recovered into 96 -well microtiter plates for absorbance measurements at $520 \mathrm{~nm}$. Quantification was based on a standard curve of 0 to $100 \mu \mathrm{M}$ pure proline (Fisher BioReagents, BP392-100). Assays were performed with replicates $804(n=3)$.

\section{Aggregate phenotypic scores (APS)}

Values from each parameter were transformed into relative values on a scale of 1 to 10 across the RIL population. The highest value was set to a score $=10$, while the lowest value was set at score $=1$ according: $y=1+(x-A) \times(10-1) / B-A$, where $y=$ normalized value, $x=$ raw value,$A=$ minimum, and $B=$ maximum. Scores in each genotype were combined into a single normalized phenotypic score and compared to SES. Scoring matrix across the RILs was analyzed by hierarchical clustering using the 'pvclust' package in R (Suzuki and Shimodaira, 2006).

\section{8 (sensitive RIL), and FL499 (super-sensitive RIL) were subjected to digital growth}

819 profiling under control and stress with the LemnaTec Scanalyzer 3D platform at the 
821 Murashige-Skoog (MS) agar for five days. Seedlings were sown in hydroponics

822 consisting of Turface MVP® submerged in Yoshida solution (Yoshida et al., 1971).

823 Plants were loaded onto the LemnaTec Scanalyzer 3D platform after 14-days. Salinity

824 treatment was introduced by adding $\mathrm{NaCl}\left(270 \mathrm{mM} \mathrm{NaCl}: 9.9 \mathrm{mM} \mathrm{CaCl}_{2}\right)$ to $\mathrm{EC}=4.5$

$825 \mathrm{dS} / \mathrm{m}(45 \mathrm{mM})$. This initial salt treatment was escalated the next day to $\mathrm{EC}=9 \mathrm{dS} / \mathrm{m}$

$826(90 \mathrm{mM})$ and maintained at that level for the duration of the experiment.

827 Solutions were changed every other day to maintain nutrient content and salinity.

828 Plants were digitally imaged daily for eighteen (18) days with an RGB and hyperspectral

829 camera. The RGB images were analyzed through a Matlab application, and

830 Phenolmage, (University of Nebraska-Lincoln). Plant size was initially reported as the

831 number of pixel squares, which were then converted into $\mathrm{cm}^{2}$ using the Fiji software

832 (Schindelin et al., 2012). Plant height was reported as measured by Phenolmage.

833 Hyperspectral image variances were computed using Matlab.

\section{Metabolite profiling by LC-MS/MS}

Shotgun metabolite analysis was performed by liquid chromatography with

837 tandem mass spectrometry (LC-MS/MS) at the Texas Tech University Center for

838 Biotechnology and Genomics. Pulverized leaf samples (100 mg) was homogenized with

839 chilled chloroform:methanol:water (1:2.5:1 [v/v/v]) and used for the separation of

840 aqueous and lipid phases. Samples were resuspended in $0.1 \%$ formic acid (v/v) and 5

$841 \mu$ aliquots were used for LC-ESI-MS/MS using the Dionex Ultimate 3000 nano-LC

842 (Thermo Scientific, San Jose, CA) interfaced to Q Exactive ${ }^{T M}$ HF Hybrid Quadrupole-

843 Orbitrap $^{\mathrm{TM}}$ mass spectrometer (Thermo Scientific, San Jose, CA). Metabolites were

844 separated on a C18 Acclaim PepMap RSLC column (Thermo Scientific, San Jose, CA)

845 at constant flow of $0.3 \mu \mathrm{L} / \mathrm{min}$ with mobile phase solvent-A ( $97.9 \%$ water/2\% ACN/0.1\%

846 FA) and solvent-B (99.9\% ACN/0.1\% FA). Metabolites were separated by following the

847 gradient of solvent-B. For MS/MS analysis, the first scan was $50-500 \mathrm{~m} / \mathrm{z}$ at mass

848 resolution of 120,000 . The ten most intense ions from the first scan were used for HCD

849 MS/MS with elevated collision energy of 20-60\% in positive and negative modes.

850 The Compound Discoverer v3.0 (Thermo Scientific) was used to detect

851 compounds with "Predicted Formula", followed by automatic online library search 
852 against the mzCloud and ChemSpider databases (Pence and Williams, 2010; Mistrik,

853 2018). Compounds from mzCloud library was checked using the mirror plot of MS/MS 854 spectra in library standards. For quantitative analysis, area under peak was calculated 855 with: precursor ion mass tolerance $=5 \mathrm{ppm}$, intensity tolerance $=30 \%$, minimum peak 856 intensity $=1 \times 10^{6}$, alignment mass tolerance $=5 \mathrm{ppm}$, peak alignment maximum shift $=$ $8572 \mathrm{~min}$, and ass tolerance of fragment ion= $10 \mathrm{ppm}$.

RNA-Seq and transcriptional network modeling

Total RNA was extracted from frozen leaf tissues using miRVana ${ }^{\mathrm{TM}}$ miRNA Isolation Kit (Invitrogen, Carlsbad, CA) and used to construct time-course (0, 24, 48, 72, $144 \mathrm{hr}$ ) RNA-Seq libraries. Strand-specific 150-bp paired-end RNA-Seq libraries were sequenced on Illumina HiSeq3000 with two replicates (Oklahoma Medical Research Foundation, OK). RNA-Seq data were analyzed using the established pipeline (Kitazumi et al., 2018). Sequence output from indexed RNA-Seq libraries were preprocessed with Cutadapt (Martin, 2011) and mapped against the Kasalath reference (Sakai et al., 2013) using HISAT2 (Kim et al., 2015). Transcript read counts were normalized by TMM (Trimmed Mean M-values) and differential expression was examined by edgeR using a false detection rate of 0.05 (McCarthy et al., 2012). 'MBCluster.Seq' package in R (Si et al., 2013). Transcript abundance were investigated by the mutual rank (MR) method (Obayashi and Kinoshita, 2009). Pearson's correlation coefficient (PCC) matrix was established for each cluster, and genes with PCC < 0.95 were excluded. MR was calculated through: $M R_{A B}=$ Square-root of $\operatorname{Rank}_{A}$ to $B \times \operatorname{Rank}_{B}$

875 to $A$. Gene interactions with values < 10 were excluded. Genetic networks were constructed and visualized using Cytoscape (Smoot et al., 2010). The annotation applied to RNA-Seq dataset was based on UniProt with additional expansion from other

878 relevant databases (The UniProt Consortium, 2018). Metabolic pathway status modeling was aided by the Kyoto Encyclopedia of Genes and Genomes (KEGG)

880 (Kanehisa and Goto, 2000; Kanehisa et al., 2018). 


\section{Statistical analysis}

Statistical analyses were conducted with R 3.5.2 (R Core Team, 2020).

Pearson's correlation coefficient matrices, gene ranks, and mutual ranks for genetic network and PCA were scripted in R-package. Tukey's HSD post-hoc tests following ANOVA were performed with the 'agricolae' package (de Mendiburu and de Mendiburu, 2019). K-means clustering was through the K-means++ code included in 'mytools' package (Yasumoto, 2020). Data visualization were through the 'ggplot2' package (Wickham and Chang, 2008).

\section{Accession Numbers}

The RNA-Seq data used in this manuscript are publicly available in the NCBI Short Read Archive (PRJNA378253:SRR11528269-SRR11528295).

\section{Acknowledgements}

This work was supported by NSF-IOS Plant Genome Research Program Grant1602494 and Bayer CropScience Endowed Professorship. Genomic computations were performed using the supercomputing facilities at the ROIS National Institute of Genetics, Mishima, Japan, and Texas Tech University High-Performance Computing Cluster. Metabolomic experiments were performed at the Texas Tech University Center for Biotechnology and Genomics. Digital phenotyping was performed using the LemnaTec

902 facilities at the University of Nebraska-Lincoln. We thank Dr. Gurdev S. Khush for his 903 valuable suggestions on the manuscript. We dedicate this work to the late Dr. Darshan

904 S. Brar, whose far-reaching vision and ingenuity served as inspiration to this project.

\section{Author Contributions}

ICMP and BGDR designed the experiments and wrote the manuscript. ICMP, $A K, K R C, B P, M Z M$, and HW performed the experiments and analyzed the data. RKS 
Table 1. Summary of the Standard Evaluation Scores (SES) and qualitative phenotypic ranking at tillering stage (V4 to V8) across the $67 \mathrm{~F}_{8}$-RILs (i.e., representative group; $n=8$ ).

\begin{tabular}{|c|c|c|c|c|c|c|c|c|c|}
\hline Genotype & $\begin{array}{c}\text { SES } \\
\text { EC=12 } \\
\text { (TTU) }\end{array}$ & Classification & $\begin{array}{l}\text { Saltol Peak } \\
\text { Marker (+/-) }\end{array}$ & $\begin{array}{c}\text { Saltol Flanking } \\
\text { markers (+/-) }\end{array}$ & Genotype & $\begin{array}{c}\text { SES } \\
E C=12 \\
\text { (TTU) }\end{array}$ & Classification & $\begin{array}{l}\text { Saltol Peak } \\
\text { Marker (+/-) }\end{array}$ & $\begin{array}{c}\text { Saltol Flanking } \\
\text { markers (+/-) }\end{array}$ \\
\hline FL301 & 4.5 & & + & - & FL460 & 5.5 & & - & - \\
\hline FL302 & 4 & Moderate & + & NA & FL463 & 5 & & - & - \\
\hline FL303 & 4.5 & Sensitive & - & NA & FL468 & 7 & & - & - \\
\hline FL327 & 5.5 & Sensitive & + & + & FL469 & 3.5 & Moderate & - & - \\
\hline FL333 & 4.5 & Sensitive & + & + & FL472 & 7 & & NA & + \\
\hline FL352 & 5 & Sensitive & - & - & FL473 & 4.5 & & + & + \\
\hline FL358 & 7 & Sensitive & + & NA & FL478 & 3 & Moderate & + & - \\
\hline FL378 & 2.5 & Tolerant & + & + & FL483 & 4.5 & & - & NA \\
\hline FL382 & 3.5 & Moderate & - & NA & FL494 & 3 & Moderate & - & - \\
\hline FL387 & 4.5 & Moderate & + & + & FL496 & 2 & Tolerant & + & NA \\
\hline FL389 & 6 & & - & - & FL499 & 9 & Supersensitive & NA & + \\
\hline FL395 & 6 & Sensitive & - & - & FL501 & 2 & Tolerant & + & + \\
\hline FL396 & 3.5 & Moderate & + & NA & FL502 & 3 & Moderate & - & - \\
\hline FL397 & 4 & & - & + & FL507 & 7 & & + & + \\
\hline FL407 & 2 & Tolerant & + & + & FL510 & 1.5 & Supertolerant & + & - \\
\hline FL409 & 6 & & - & - & FL512 & 8 & & - & - \\
\hline FL416 & 2.5 & Tolerant & NA & + & FL530 & 3 & Moderate & + & + \\
\hline FL418 & 7 & & - & - & FL541 & 4 & & + & + \\
\hline FL428 & 2 & Tolerant & + & - & FL542 & 7 & & + & + \\
\hline FL431 & 4.5 & Sensitive & - & - & FL545 & 4 & Moderate & + & - \\
\hline FL433 & 4 & & - & - & FL557 & 3.5 & Moderate & - & - \\
\hline FL434 & 4.5 & & - & NA & FL560 & 2.5 & Tolerant & + & + \\
\hline FL435 & 5 & & - & - & FL561 & 6 & Sensitive & + & + \\
\hline FL441 & 7 & & - & + & FL567 & 4 & Moderate & - & - \\
\hline FL442 & 7 & & + & - & FL568 & 4 & Moderate & - & NA \\
\hline FL443 & 7 & & NA & - & FL572 & 6 & & - & - \\
\hline FL445 & 7 & & + & + & FL577 & 6 & & + & NA \\
\hline FL446 & 5 & & - & + & FL588 & 6 & & + & NA \\
\hline FL448 & 4 & Moderate & - & - & FL590 & 6 & & + & NA \\
\hline FL449 & 2.5 & Tolerant & + & - & IR29 & 8 & Parent (Sensitive) & - & - \\
\hline
\end{tabular}

913 Salt tolerance was compared with Saltol genotype, which was determined based on the presence of the Pokkali allele at the peak and/or 914 flanking markers (Thomson et al., 2010). 
bioRxiv preprint doi: https://doi.org/10.1101/2020.06.25.171603; this version posted June 26, 2020. The copyright holder for this preprint (which was not certified by peer review) is the author/funder. All rights reserved. No reuse allowed without permission.

915

916 


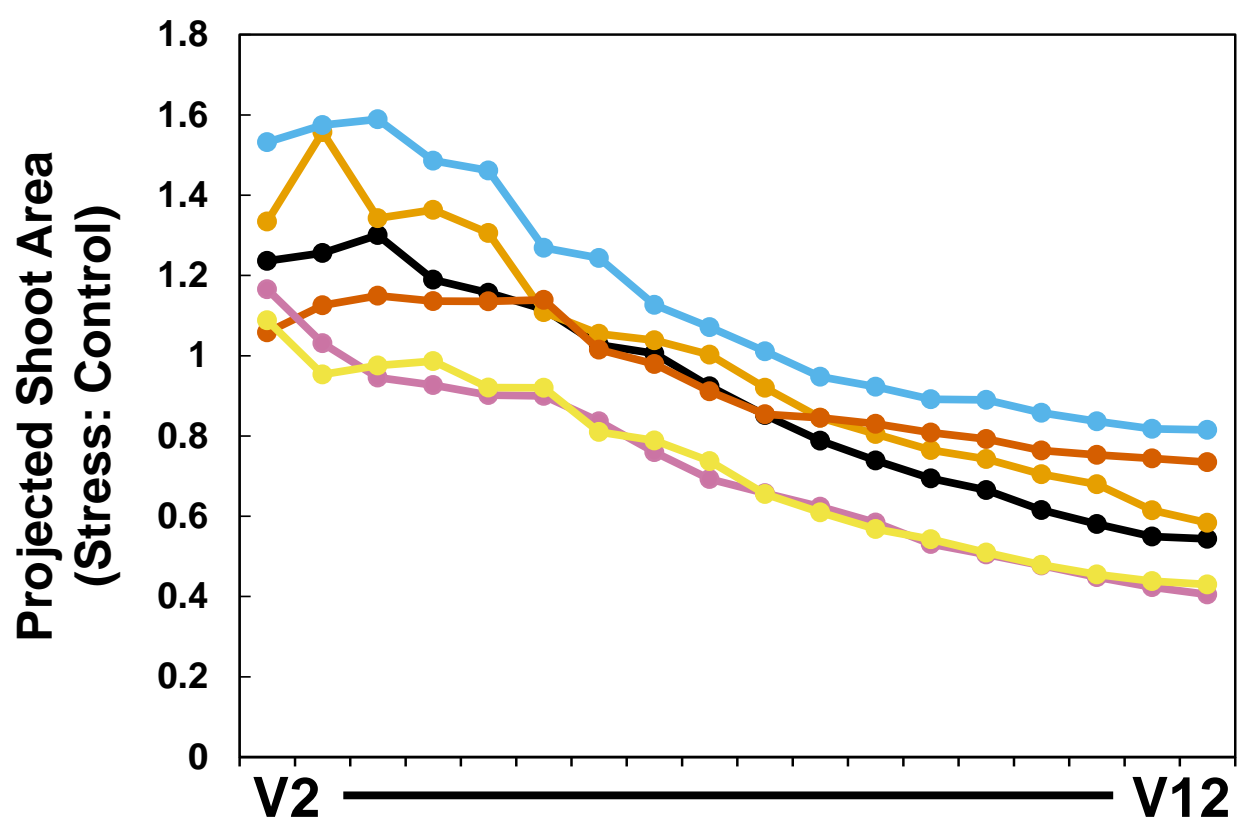

- IR29

18 days

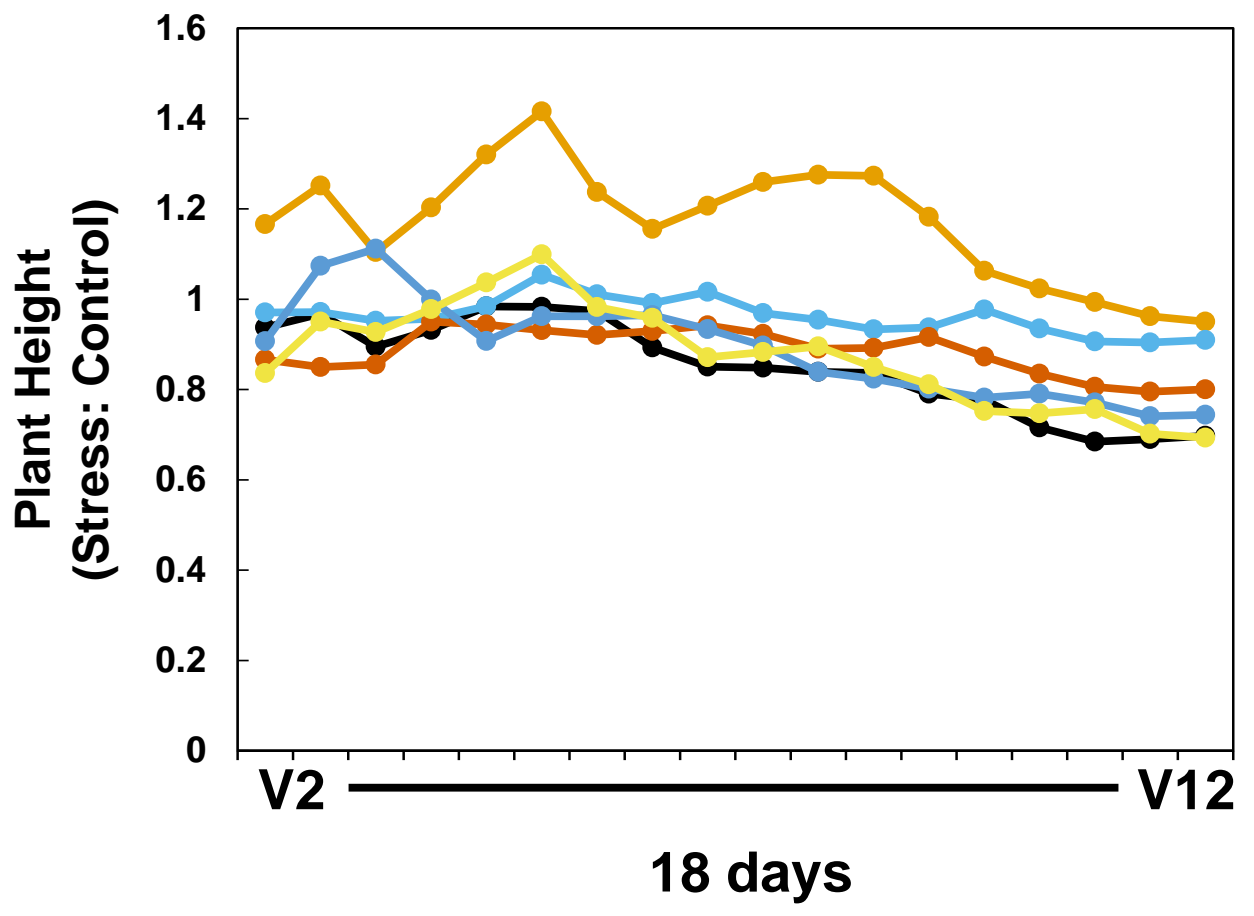

Pokkali

FL478

FL510

FL454

FL499

Supplemental Figure 1. Stress to control ratios of projected shoot area (PSA) and plant height among the different genotypes used in the study across the real-time imaging period. Ratios of stress and control measurements of PSA and plant height were plotted for all the genotypes used in the study in the same time frame as Figure 1. This was used to assess the extent of growth penalty incurred by each genotype through the stress period. Ratio values greater than 1 indicate a larger value for the stress treatment compared to the control, while those lower than 1 indicate a reduction in growth relative to the control. 

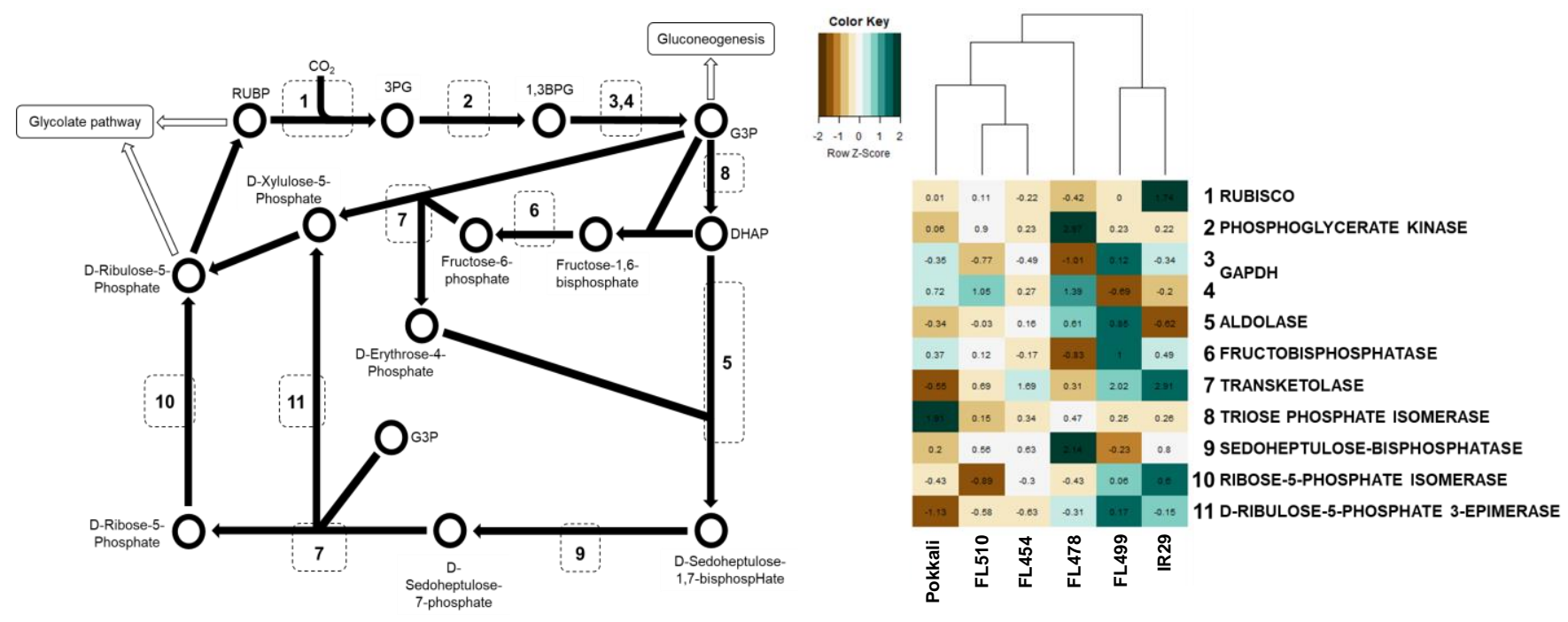

Supplemental Figure 3. Pathway induction heatmaps under stress for genes in the Calvin cycle. Values were generated from the fold-change under control conditions and the stress time point with the widest margin from control. The genotypes were hierarchically clustered to show similarity in pathway induction. RuBP = Ribulose 1,5-bisphosphate; RUBISCO = Ribulose-1,5-bisphosphate carboxylase/oxygenase; 1,3-BPG = 1,3-bisphosphoglycerate; $P P K=$ Phosphoglycerate kinase; 3-PG = 3phosphoglycerate; GAPDH = Glyceraldehyde-3-phosphate dehydrogenase; GAP = Glyceraldehyde-3phosphate; TPI = Triose-phosphate isomerase; DHAP = Dihydroxyacetone phosphate; G3P = Glyceraldehyde 3-phosphate. 
Supplemental Table 1. $R^{2}$ and P-values of different traits tested in the phenotyping of the IR29 $x$ Pokkali $F$

\begin{tabular}{lcc}
\hline \multicolumn{1}{c}{ Trait } & $\mathbf{R}^{2}$ value & $\mathbf{p}$-value \\
\hline ELI at 144H & 0.001 & 0.85 \\
ELI Ratio from 72 to 144 $\mathrm{h}$ & 0.017 & 0.43 \\
Root biomass ratio & 0.031 & 0.29 \\
Shoot biomass ratio & 0.00029 & 0.92 \\
Stomatal Conductance & 0.021926 & 0.38 \\
Chlorophyll & 0.003294 & 0.73 \\
Na/K ratio at 144h & 0.147647 & $0.02^{*}$ \\
Na/K slope change from $0 \mathrm{H}$ to $144 \mathrm{~h}$ & 0.036607 & 0.25 \\
Na ratio from stress to control & $5.06 \mathrm{E}-05$ & 0.97 \\
K ratio from stress to control & 0.047644 & 0.19 \\
Peroxidase activity at $0 \mathrm{~h}$ & 0.001414 & 0.82 \\
Peroxidase activity at $144 \mathrm{~h}$ & 0.169299 & $0.01^{*}$ \\
Peroxidase activity ratio from 0 to 144 $\mathrm{h}$ & 0.113259 & $0.04^{*}$ \\
Lipid peroxidation at $0 \mathrm{~h}$ & 0.028629 & 0.31 \\
Lipid peroxidation at $144 \mathrm{~h}$ & 0.01976 & 0.41 \\
Lipid peroxidation ratio from stress to control & 0.010532 & 0.55 \\
\hline
\end{tabular}


bioRxiv preprint doi: https://doi.org/10.1101/2020.06.25.171603; this version posted June 26, 2020. The copyright holder for this preprint (which was not certified by peer review) is the author/funder. All rights reserved. No reuse allowed without permission.

IL population relative to SES. Traits with significant relationship with SES based on regression analysis $(P<0.05)$ a 
bioRxiv preprint doi: https://doi.org/10.1101/2020.06.25.171603; this version posted June 26, 2020. The copyright holder for this preprint (which was not certified by peer review) is the author/funder. All rights reserved. No reuse allowed without permission.

are marked with an asterisk $\left(^{*}\right)$. 
Supplemental Table 2. Summary and mapping statistics of the RNA-seq libraries constructed for the parental

\begin{tabular}{|c|c|c|c|c|c|c|c|}
\hline \multirow{2}{*}{ Sample } & \multirow{2}{*}{ Replicate } & \multicolumn{6}{|c|}{ Number of Paired Reads } \\
\hline & & IR29 & Pokkali & FL478 & FL510 & FL499 & FL454 \\
\hline $\mathrm{Oh}$ & $A$ & $16,736,902$ & $21,972,858$ & $19,519,378$ & $27,741,440$ & $25,303,994$ & $22,958,328$ \\
\hline $\mathrm{Oh}$ & B & $23,566,931$ & $31,223,873$ & $27,374,472$ & $31,095,774$ & $28,887,331$ & $32,035,095$ \\
\hline $24 \mathrm{~h}$ & $A$ & $17,387,989$ & $18,977,029$ & $16,264,496$ & $30,592,532$ & $25,009,427$ & $17,272,958$ \\
\hline $24 \mathrm{~h}$ & B & $24,939,854$ & $27,237,708$ & $23,010,419$ & $34,146,243$ & $28,503,630$ & $24,233,186$ \\
\hline $48 \mathrm{~h}$ & A & $31,555,196$ & $26,903,719$ & $28,145,394$ & $31,562,536$ & $27,070,597$ & $31,891,918$ \\
\hline $48 \mathrm{~h}$ & $\mathrm{~B}$ & $33,789,866$ & $30,030,083$ & $30,990,592$ & $34,024,720$ & $30,210,218$ & $34,196,118$ \\
\hline $72 \mathrm{~h}$ & $A$ & $25,291,270$ & $30,502,192$ & $27,587,928$ & $26,650,239$ & $25,432,219$ & $30,055,914$ \\
\hline $72 \mathrm{~h}$ & B & $34,825,718$ & $33,309,312$ & $29,991,355$ & $28,917,995$ & $28,184,071$ & $32,004,826$ \\
\hline $144 \mathrm{~h}$ & A & $26,521,109$ & $32,068,870$ & $31,701,845$ & $28,877,883$ & $31,308,395$ & $34,820,920$ \\
\hline $144 \mathrm{~h}$ & $B$ & $36,076,103$ & $35,384,106$ & $33,676,271$ & $31,871,680$ & $35,266,910$ & $37,216,213$ \\
\hline \multirow{2}{*}{ Sample } & \multirow{2}{*}{ Replicate } & \multicolumn{6}{|c|}{ Map Rates } \\
\hline & & IR29 & Pokkali & FL478 & FL510 & FL499 & FL454 \\
\hline $\mathrm{Oh}$ & $A$ & $80.54 \%$ & $73.60 \%$ & $83.83 \%$ & $92.98 \%$ & $93.03 \%$ & $91.33 \%$ \\
\hline $\mathrm{Oh}$ & B & $78.62 \%$ & $71.49 \%$ & $81.77 \%$ & $90.12 \%$ & $90.12 \%$ & $88.73 \%$ \\
\hline $24 \mathrm{~h}$ & A & $68.20 \%$ & $82.29 \%$ & $63.37 \%$ & $93.90 \%$ & $93.72 \%$ & $86.42 \%$ \\
\hline $24 \mathrm{~h}$ & B & $67.39 \%$ & $80.88 \%$ & $62.66 \%$ & $92.03 \%$ & $92.32 \%$ & $84.28 \%$ \\
\hline $48 \mathrm{~h}$ & $A$ & $94.33 \%$ & $94.60 \%$ & $94.57 \%$ & $94.30 \%$ & $94.54 \%$ & $94.50 \%$ \\
\hline $48 \mathrm{~h}$ & B & $92.69 \%$ & $93.38 \%$ & $93.31 \%$ & $92.76 \%$ & $93.39 \%$ & $92.99 \%$ \\
\hline $72 \mathrm{~h}$ & A & $90.92 \%$ & $94.07 \%$ & $94.29 \%$ & $94.24 \%$ & $94.06 \%$ & $93.77 \%$ \\
\hline $72 \mathrm{~h}$ & B & $89.27 \%$ & $92.39 \%$ & $93.08 \%$ & $93.11 \%$ & $92.78 \%$ & $92.01 \%$ \\
\hline $144 \mathrm{~h}$ & A & $90.69 \%$ & $93.14 \%$ & $91.68 \%$ & $93.97 \%$ & $94.49 \%$ & $93.14 \%$ \\
\hline $144 \mathrm{~h}$ & $B$ & $89.44 \%$ & $91.81 \%$ & $90.52 \%$ & $93.10 \%$ & $93.56 \%$ & $91.85 \%$ \\
\hline
\end{tabular}


bioRxiv preprint doi: https://doi.org/10.1101/2020.06.25.171603; this version posted June 26, 2020. The copyright holder for this preprint (which was not certified by peer review) is the author/funder. All rights reserved. No reuse allowed without permission.

genotypes and selected RILs. 


\section{Parsed Citations}

AOAC. (1990). Official methods of analysis. Assoc Anal Chem

Pubmed: Author and Title

Google Scholar: Author Only Title Only Author and Title

Ashikari, M., and Matsuoka, M. (2006). Identification, isolation and pyramiding of quantitative trait loci for rice breeding. Trends in Plant Science 11, 344-350.

Pubmed: Author and Title

Google Scholar: Author Only Title Only Author and Title

Ashraf, M., and Foolad, M.R. (2007). Roles of glycine betaine and proline in improving plant abiotic stress resistance. Environ. Exp. Bot. 59, 206-216.

Pubmed: Author and Title

Google Scholar: Author Only Title Only Author and Title

Babu, N.N., Krishnan, S.G., Vinod, K.K., Krishnamurthy, S.L., Singh, V.K., Singh, M.P., Singh, R., Ellur, R.K., Rai, V., and Bollinedi, H. (2017). Marker aided incorporation of Saltol, a major QTL associated with seedling stage salt tolerance, into Oryza sativa 'Pusa basmati 1121'. Front. Plant Sci. 8, 41.

Pubmed: Author and Title

Google Scholar: Author Only Title Only Author and Title

Ballou, S.M., Yun, K.-Y., Cheng, C., and de los Reyes, B.G. (2007). Cold sensitivity gradient in tuber-bearing Solanum based on physiological and transcript profiles. Crop Sci. 47, 2027-2035.

Pubmed: Author and Title

Google Scholar: Author Only Title Only Author and Title

Bates, L.S., Waldren, R.P., and Teare, I.D. (1973). Rapid determination of free proline for water-stress studies. Plant Soil 39, 205-207.

Pubmed: Author and Title

Google Scholar: Author Only Title Only Author and Title

Bimpong, I.K., Manneh, B., Sock, M., Diaw, F., Amoah, N.K.A, Ismail, AM., Gregorio, G., Singh, R.K., and Wopereis, M. (2016). Improving salt tolerance of lowland rice cultivar 'Rassi'through marker-aided backcross breeding in West Africa. Plant Sci. 242, 288-299.

Pubmed: Author and Title

Google Scholar: Author Only Title Only Author and Title

Birchler, J.A, Yao, H., Chudalayandi, S., Vaiman, D., and Veitia, R.A (2010). Heterosis. Plant Cell 22, 2105-2112.

Pubmed: Author and Title

Google Scholar: Author Only Title Only Author and Title

Bomblies, K., and Weigel, D. (2007). Hybrid necrosis: autoimmunity as a potential gene-flow barrier in plant species. Nat. Rev. Genet. 8, 382-393.

Pubmed: Author and Title

Google Scholar: Author Only Title Only Author and Title

Bonilla, P., Dvorak, J., Mackell, D., Deal, K., and Gregorio, G. (2002). RFLP and SSLP mapping of salinity tolerance genes in chromosome 1 of rice (Oryza sativa L.) using recombinant inbred lines. Philipp. Agric. Sci. (Philippines).

Pubmed: Author and Title

Google Scholar: Author Only Title Only Author and Title

Boyle, E.A, Li, Y.I., and Pritchard, J.K. (2017). An expanded view of complex traits: from polygenic to omnigenic. Cell 169, $1177-1186$. Pubmed: Author and Title

Google Scholar: Author Only Title Only Author and Title

Campbell, M.T., Knecht, AC., Berger, B., Brien, C.J., Wang, D., and Walia, H. (2015). Integrating Image-Based Phenomics and Association Analysis to Dissect the Genetic Architecture of Temporal Salinity Responses in Rice. Plant Physiol. 168, $1476-1489$.

Pubmed: Author and Title

Google Scholar: Author Only Title Only Author and Title

Chattopadhyay, K., Nath, D., Mohanta, R.L., Bhattacharyya, S., Marndi, B.C., Nayak, AK., Singh, D.P., Sarkar, R.K., and Singh, O.N. (2014). Diversity and validation of microsatellite markers in'Saltol'QTL region in contrasting rice genotypes for salt tolerance at the early vegetative stage. Aust. J. Crop Sci. 8, 356.

Pubmed: Author and Title

Google Scholar: Author Only Title Only Author and Title

Chen, Y., Xu, Y., Luo, W., Li, W., Chen, N., Zhang, D., and Chong, K. (2013). The F-Box Protein OsFBK12 Targets OsSAMS1 for

Degradation and Affects Pleiotropic Phenotypes, Including Leaf Senescence, in Rice. Plant Physiol. 163, 1673.

Pubmed: Author and Title

Google Scholar: Author Only Title Only Author and Title

Claussen, W. (2005). Proline as a measure of stress in tomato plants. Plant Sci. 168, 241-248.

Pubmed: Author and Title

Google Scholar: Author Only Title Only Author and Title 
Collard, B.C., and Mackill, D.J. (2008). Marker-assisted selection: an approach for precision plant breeding in the twenty-first century.

Philos. T. R. Soc. B. 363, 557-572.

Pubmed: Author and Title

Google Scholar: Author Only Title Only Author and Title

Cosio, C., and Dunand, C. (2008). Specific functions of individual class III peroxidase genes. J. Exp. Bot. 60, $391-408$.

Pubmed: Author and Title

Google Scholar: Author Only Title Only Author and Title

Counce, P.A, Keisling, T.C., and Mitchell, AJ. (2000). A uniform, objective, and adaptive system for expressing rice development. Crop Science 40, 436-443.

Pubmed: Author and Title

Google Scholar: Author Only Title Only Author and Title

de los Reyes, B.G. (2019). Genomic and epigenomic bases of transgressive segregation - New breeding paradigm for novel plant phenotypes. Plant Sci. 288, 110213.

Pubmed: Author and Title

Google Scholar: Author Only Title Only Author and Title

de los Reyes, B.G., Yun, S.J., Herath, V., Xu, F., Park, M.R., Lee, J.I., and Kim, K.Y. (2013). Phenotypic, Physiological, and Molecular Evaluation of Rice Chilling Stress Response at the Vegetative Stage. In Rice Protocols, Y. Yang, ed (Totowa, NJ: Humana Press), pp. 227-241.

Pubmed: Author and Title

Google Scholar: Author Only Title Only Author and Title

de los Reyes, B.G., Kim, Y.S., Mohanty, B., Kumar, A, Kitazumi, A, Pabuayon, I.C.M., Sandhu, N., and Lee, D.-Y. (2018). Cold and Water Deficit Regulatory Mechanisms in Rice: Optimizing Stress Tolerance Potential by Pathway Integration and Network Engineering. In Rice Genomics, Genetics and Breeding, T. Sasaki and M. Ashikari, eds (Singapore: Springer Singapore), pp. $317-359$.

Pubmed: Author and Title

Google Scholar: Author Only Title Only Author and Title

de Mendiburu, F., and de Mendiburu, M.F. (2019). Package 'agricolae'. R package version, 1.2-8.

Pubmed: Author and Title

Google Scholar: Author Only Title Only Author and Title

Deng, X., Yuan, S., Cao, H., Lam, S.M., Shui, G., Hong, Y., and Wang, X. (2019). Phosphatidylinositol-hydrolyzing phospholipase C4 modulates rice response to salt and drought. Plant Cell Environ. 42, 536-548.

Pubmed: Author and Title

Google Scholar: Author Only Title Only Author and Title

deVicente, M.C., and Tanksley, S.D. (1993). QTL analysis of transgressive segregation in an interspecific tomato cross. Genetics 134, 585-596.

Pubmed: Author and Title

Google Scholar: Author Only Title Only Author and Title

Dickinson, H.G., Hiscock, S.J., Crane, P.R., Rieseberg, L.H., Widmer, A, Arntz, AM., and Burke, B. (2003). The genetic architecture necessary for transgressive segregation is common in both natural and domesticated populations. Philos T. R. Soc. B. 358, $1141-1147$. Pubmed: Author and Title

Google Scholar: Author Only Title Only Author and Title

Dittrich-Reed, D.R., and Fitzpatrick, B.M. (2013). Transgressive Hybrids as Hopeful Monsters. Evol. Biol. 40, $310-315$.

Pubmed: Author and Title

Google Scholar: Author Only Title Only Author and Title

Draeger, C., Ndinyanka Fabrice, T., Gineau, E., Mouille, G., Kuhn, B.M., Moller, I., Abdou, M.-T., Frey, B., Pauly, M., Bacic, A, and Ringli, C. (2015). Arabidopsis leucine-rich repeat extensin (LRX) proteins modify cell wall composition and influence plant growth. BMC Plant Biol. 15, 155.

Pubmed: Author and Title

Google Scholar: Author Only Title Only Author and Title

Eshed, Y., and Zamir, D. (1995). An introgression line population of Lycopersicon pennellii in the cultivated tomato enables the identification and fine mapping of yield-associated QTL. Genetics 141, 1147-1162.

Pubmed: Author and Title

Google Scholar: Author Only Title Only Author and Title

Gendron, J.M. (2019). Genetic Network Rewiring Configures ANovel Dehydration Stress Tolerant Phenotype In Transgressive Recombinant Inbreds of Rice (Oryza sativa L., indica), B.G. de los Reyes, G.B. Burow, and R. Shim, eds.

Pubmed: Author and Title

Google Scholar: Author Only Title Only Author and Title

Gregorio, G.B., Senadhira, D., and Mendoza, R.D. (1997). Screening rice for salinity tolerance (IRRI discussion paper series).

Gregorio, G.B., Senadhira, D., Mendoza, R.D., Manigbas, N.L., Roxas, J.P., and Guerta, C.Q. (2002). Progress in breeding for salinity tolerance and associated abiotic stresses in rice. Field Crops Res. 76, 91-101.

Pubmed: Author and Title 
Google Scholar: Author Only Title Only Author and Title

Huyen, L.T.N., Cuc, L.M., Ismail, AM., and Ham, L.H. (2012). Introgression the Salinity Tolerance QTLs SalTol into AS996, the Elite Rice Variety of Vietnam Am J. Plant Sci. 3, 981-987.

Pubmed: Author and Title

Google Scholar: Author Only Title Only Author and Title

Huyen, L.T.N., Cuc, L.M., Ham, L.H., and Khanh, T.D. (2013). Introgression the SALTOL QTL into Q5DB, the elite variety of Vietnam using marker-assisted-selection (MAS). Am. J. BioSci. 1, 80À84.

Pubmed: Author and Title

Google Scholar: Author Only Title Only Author and Title

Islam, M.R., Gregorio, G.B., Salam, M.A, Collard, B.C., Singh, R.K., and Hassan, L. (2012). Validation of SalTol linked markers and haplotype diversity on chromosome 1 of rice. Mol. Plant Breed. 3.

Pubmed: Author and Title

Google Scholar: Author Only Title Only Author and Title

Jambunathan, N. (2010). Determination and Detection of Reactive Oxygen Species (ROS), Lipid Peroxidation, and Electrolyte Leakage in Plants. In Plant Stress Tolerance: Methods and Protocols, R. Sunkar, ed (Totowa, NJ: Humana Press), pp. $291-297$.

Pubmed: Author and Title

Google Scholar: Author Only Title Only Author and Title

Kand'ár, R., and Žáková, P. (2008). Allantoin as a marker of oxidative stress in human erythrocytes. Clin. Chem. Lab. Med. 46, $1270-1274$.

Pubmed: Author and Title

Google Scholar: Author Only Title Only Author and Title

Kanehisa, M., and Goto, S. (2000). KEGG: Kyoto Encyclopedia of Genes and Genomes. Nucleic Acids Res. $28,27-30$.

Pubmed: Author and Title

Google Scholar: Author Only Title Only Author and Title

Kanehisa, M., Sato, Y., Furumichi, M., Morishima, K., and Tanabe, M. (2018). New approach for understanding genome variations in KEGG. Nucleic Acids Res. 47, D590-D595.

Pubmed: Author and Title

Google Scholar: Author Only Title Only Author and Title

Khush, G.S. (2001). Green revolution: the way forward. Nat. Rev. Genet. 2, 815-822.

Pubmed: Author and Title

Google Scholar: Author Only Title Only Author and Title

Khush, G.S. (2005). What it will take to feed 5.0 billion rice consumers in 2030. Plant Mol. Biol. 59, 1-6.

Pubmed: Author and Title

Google Scholar: Author Only Title Only Author and Title

Kim, D., Langmead, B., and Salzberg, S.L. (2015). HISAT: a fast spliced aligner with low memory requirements. Nat. Methods $12,357-360$.

Pubmed: Author and Title

Google Scholar: Author Only Title Only Author and Title

Kitazumi, A, Pabuayon, I.C.M., Ohyanagi, H., Fujita, M., Osti, B., Shenton, M.R., Kakei, Y., Nakamura, Y., Brar, D.S., Kurata, N., and de los Reyes, B.G. (2018). Potential of Oryza officinalis to augment the cold tolerance genetic mechanisms of Oryza sativa by network complementation. Sci. Rep. 8, 16346.

Pubmed: Author and Title

Google Scholar: Author Only Title Only Author and Title

Kitomi, Y., Inahashi, H., Takehisa, H., Sato, Y., and Inukai, Y. (2012). OsIAA13-mediated auxin signaling is involved in lateral root initiation in rice. Plant Sci. 190, 116-122.

Pubmed: Author and Title

Google Scholar: Author Only Title Only Author and Title

Kosugi, S., and Ohashi, Y. (2002). E2F sites that can interact with E2F proteins cloned from rice are required for meristematic tissuespecific expression of rice and tobacco proliferating cell nuclear antigen promoters. Plant J. 29, 45-59.

Pubmed: Author and Title

Google Scholar: Author Only Title Only Author and Title

Kumar, A, Sandhu, N., Dixit, S., Yadav, S., Swamy, B.P.M., and Shamsudin, N.AA (2018). Marker-assisted selection strategy to pyramid two or more QTLs for quantitative trait-grain yield under drought. Rice 11, 35.

Pubmed: Author and Title

Google Scholar: Author Only Title Only Author and Title

Kumar, G., Kushwaha, H.R., Panjabi-Sabharwal, V., Kumari, S., Joshi, R., Karan, R., Mittal, S., Pareek, S.L.S., and Pareek, A (2012). Clustered metallothionein genes are co-regulated in rice and ectopic expression of OsMT1e-Pconfers multiple abiotic stress tolerance in tobacco via ROS scavenging. BMC Plant Biol. 12, 107.

Pubmed: Author and Title

Google Scholar: Author Only Title Only Author and Title

Kumar, V., Shriram, V., Kishor, P.K., Jawali, N., and Shitole, M. (2010). Enhanced proline accumulation and salt stress tolerance of 
transgenic indica rice by over-expressing P5CSF129A gene. Plant Biotechnol. Rep. 4, 37-48.

Pubmed: Author and Title

Google Scholar: Author Only Title Only Author and Title

Kuramata, M., Masuya, S., Takahashi, Y., Kitagawa, E., Inoue, C., Ishikawa, S., Youssefian, S., and Kusano, T. (2008). Novel CysteineRich Peptides from Digitaria ciliaris and Oryza sativa Enhance Tolerance to Cadmium by Limiting its Cellular Accumulation. Plant Cell Physiol. 50, 106-117.

Pubmed: Author and Title

Google Scholar: Author Only Title Only Author and Title

La, H., Li, J., Ji, Z, Cheng, Y., Li, X., Jiang, S., Venkatesh, P.N., and Ramachandran, S. (2006). Genome-wide analysis of cyclin family in rice (Oryza Sativa L.). Mol Genet. Genomics 275, 374-386.

Pubmed: Author and Title

Google Scholar: Author Only Title Only Author and Title

Li, Z, Mu, P., Li, C., Zhang, H., Li, Z, Gao, Y., and Wang, X. (2005). QTL mapping of root traits in a doubled haploid population froma cross between upland and lowland japonica rice in three environments. Theor. Appl. Genet. 110, $1244-1252$.

Pubmed: Author and Title

Google Scholar: Author Only Title Only Author and Title

Lippman, ZB., and Zamir, D. (2007). Heterosis: revisiting the magic. Trends Genet. 23, 60-66.

Pubmed: Author and Title

Google Scholar: Author Only Title Only Author and Title

Lippman, ZB., Semel, Y., and Zamir, D. (2007). An integrated view of quantitative trait variation using tomato interspecific introgression lines. Curr. Opin. Genet. Dev. 17, 545-552.

Pubmed: Author and Title

Google Scholar: Author Only Title Only Author and Title

Liu, W., Xiang, Y., Zhang, X., Han, G., Sun, X., Sheng, Y., Yan, J., Scheller, H.V., and Zhang, A (2019a). Over-Expression of a Maize N-

Acetylglutamate Kinase Gene (ZmNAGK) Improves Drought Tolerance in Tobacco. Front. Plant Sci. 9, 1902.

Pubmed: Author and Title

Google Scholar: Author Only Title Only Author and Title

Liu, X., Li, Y.I., and Pritchard, J.K. (2019b). Trans Effects on Gene Expression Can Drive Omnigenic Inheritance. Cell 177, 10221034.e1026.

Pubmed: Author and Title

Google Scholar: Author Only Title Only Author and Title

Lv, Y., Yang, M., Hu, D., Yang, Z, Ma, S., Li, X., and Xiong, L. (2017). The OsMYB30 Transcription Factor Suppresses Cold Tolerance by Interacting with a JAZ Protein and Suppressing beta-Amylase Expression. Plant Physiol. 173, 1475-1491.

Pubmed: Author and Title

Google Scholar: Author Only Title Only Author and Title

Martin, M. (2011). Cutadapt removes adapter sequences from high-throughput sequencing reads. EMBnet J. $17,3$.

Pubmed: Author and Title

Google Scholar: Author Only Title Only Author and Title

McCarthy, D.J., Chen, Y., and Smyth, G.K. (2012). Differential expression analysis of multifactor RNASeq experiments with respect to biological variation. Nucleic Acids Res. 40, 4288-4297.

Pubmed: Author and Title

Google Scholar: Author Only Title Only Author and Title

Mistrik, R. (2018). mzCLOUD: Aspectral tree library for the Identification of" unknown unknowns". In ABSTRACTS OF PAPERS OF THE AMERICAN CHEMICAL SOCIETY (AMER CHEMICAL SOC 1155 16TH ST, NW, WASHINGTON, DC 20036 USA).

Pubmed: Author and Title

Google Scholar: Author Only Title Only Author and Title

Miyamoto, K., Shimizu, T., Mochizuki, S., Nishizawa, Y., Minami, E., Nojiri, H., Yamane, H., and Okada, K. (2013). Stress-induced expression of the transcription factor RERJ1 is tightly regulated in response to jasmonic acid accumulation in rice. Protoplasma 250, 241-249.

Pubmed: Author and Title

Google Scholar: Author Only Title Only Author and Title

Moeljopawiro, S., and Ikehashi, H. (1981). Inheritance of salt tolerance in rice. Euphytica 30, 291-300.

Pubmed: Author and Title

Google Scholar: Author Only Title Only Author and Title

Mukhopadhyay, A, Vij, S., and Tyagi, AK. (2004). Overexpression of a zinc-finger protein gene from rice confers tolerance to cold, dehydration, and salt stress in transgenic tobacco. Proc. Natl. Acad. Sci. U. S. A 101, 6309-6314.

Pubmed: Author and Title

Google Scholar: Author Only Title Only Author and Title

Munns, R., and Gilliham, M. (2015). Salinity tolerance of crops-what is the cost? New Phytol. 208, 668-673.

Pubmed: $\underline{\text { Author and Title }}$ 
Google Scholar: Author Only Title Only Author and Title

Munns, R., Day, D.A, Fricke, W., Watt, M., Arsova, B., Barkla, B.J., Bose, J., Byrt, C.S., Chen, Z-H., Foster, K.J., Gilliham, M., Henderson, S.W., Jenkins, C.L.D., Kronzucker, H.J., Miklavcic, S.J., Plett, D., Roy, S.J., Shabala, S., Shelden, M.C., Soole, K.L., Taylor, N.L., Tester, M., Wege, S., Wegner, L.H., and Tyerman, S.D. (2020). Energy costs of salt tolerance in crop plants. New Phytol. 225, $1072-1090$.

Pubmed: Author and Title

Google Scholar: Author Only Title Only Author and Title

Obayashi, T., and Kinoshita, K. (2009). Rank of Correlation Coefficient as a Comparable Measure for Biological Significance of Gene Coexpression. DNARes. 16, 249-260.

Pubmed: Author and Title

Google Scholar: Author Only Title Only Author and Title

Oda, Y., Kobayashi, N.I., Tanoi, K., Ma, J.F., Itou, Y., Katsuhara, M., Itou, T., and Horie, T. (2018). T-DNA tagging-based gain-of-function of OsHKT1; 4 reinforces Na exclusion from leaves and stems but triggers Na toxicity in roots of rice under salt stress. Int. J. Mol. Sci. 19, 235.

Pubmed: Author and Title

Google Scholar: Author Only Title Only Author and Title

Ogawa, S., Kawahara-Miki, R., Miyamoto, K., Yamane, H., Nojiri, H., Tsujii, Y., and Okada, K. (2017). OsMYC2 mediates numerous defence-related transcriptional changes via jasmonic acid signalling in rice. Biochem Biophys. Res. Comm 486, 796-803.

Pubmed: Author and Title

Google Scholar: Author Only Title Only Author and Title

Ohta, M., and Takaiwa, F. (2015). OsHrd3 is necessary for maintaining the quality of endoplasmic reticulum-derived protein bodies in rice endosperm. J. Exp. Bot. 66, 4585-4593.

Pubmed: Author and Title

Google Scholar: Author Only Title Only Author and Title

Ouyang, S.-Q., Liu, Y.-F., Liu, P., Lei, G., He, S.-J., Ma, B., Zhang, W.-K., Zhang, J.-S., and Chen, S.-Y. (2010). Receptor-like kinase OsSIK1 improves drought and salt stress tolerance in rice (Oryza sativa) plants. Plant J. 62, 316-329.

Pubmed: Author and Title

Google Scholar: Author Only Title Only Author and Title

Pence, H.E., and Williams, A (2010). ChemSpider: An Online Chemical Information Resource. J. Chem Educ. 87, $1123-1124$.

Pubmed: Author and Title

Google Scholar: Author Only Title Only Author and Title

Peng, S., Khush, G., and Cassman, K. (1994). Evolution of the new plant ideotype for increased yield potential. In Breaking the Yield Barrier: Proceedings of a Workshop on Rice Yield Potential in Favorable Environments. International Rice Research Institute, Los

Banos, Philippines, pp. 5-20.

Pubmed: Author and Title

Google Scholar: Author Only Title Only Author and Title

Peng, S., Khush, G.S., Virk, P., Tang, Q., and Zou, Y. (2008). Progress in ideotype breeding to increase rice yield potential. Field Crops Res. 108, 32-38.

Pubmed: Author and Title

Google Scholar: Author Only Title Only Author and Title

Perochon, A, Adon, D., Galaud, J.-P., and Ranty, B. (2011). Calmodulin and calmodulin-like proteins in plant calcium signaling.

Biochimie 93, 2048-2053.

Pubmed: Author and Title

Google Scholar: Author Only Title Only Author and Title

Pingali, P.L. (2012). Green revolution: impacts, limits, and the path ahead. Proc. Natl. Acad. Sci. U. S. A 109, $12302-12308$.

Pubmed: Author and Title

Google Scholar: Author Only Title Only Author and Title

R Core Team (2020). R: A Language and Environment for Statistical Computing (Vienna, Austria).

Ramamoorthy, R., Jiang, S.-Y., Kumar, N., Venkatesh, P.N., and Ramachandran, S. (2008). AComprehensive Transcriptional Profiling of the WRKY Gene Family in Rice Under Various Abiotic and Phytohormone Treatments. Plant Cell Physiol. 49, 865-879.

Pubmed: Author and Title

Google Scholar: Author Only Title Only Author and Title

Ren, Z-H., Gao, J.-P., Li, L.-G., Cai, X.-L., Huang, W., Chao, D.-Y., Zhu, M.-Z, Wang, Z-Y., Luan, S., and Lin, H.-X. (2005). Arice quantitative trait locus for salt tolerance encodes a sodium transporter. Nat. Genet. 37, 1141-1146.

Pubmed: Author and Title

Google Scholar: Author Only Title Only Author and Title

Rieseberg, L.H., Archer, M.A, and Wayne, R.K. (1999). Transgressive segregation, adaptation and speciation. Heredity 83, $363-372$.

Pubmed: Author and Title

Google Scholar: Author Only Title Only Author and Title

Rieseberg, L.H., Sinervo, B., Linder, C.R., Ungerer, M.C., and Arias, D.M. (1996). Role of Gene Interactions in Hybrid Speciation: 
Evidence from Ancient and Experimental Hybrids. Science 272, 741-745.

Pubmed: Author and Title

Google Scholar: Author Only Title Only Author and Title

Rieseberg, L.H., Raymond, O., Rosenthal, D.M., Lai, Z, Livingstone, K., Nakazato, T., Durphy, J.L., Schwarzbach, AE., Donovan, L.A, and Lexer, C. (2003). Major Ecological Transitions in Wild Sunflowers Facilitated by Hybridization. Science 301, $1211-1216$.

Pubmed: Author and Title

Google Scholar: Author Only Title Only Author and Title

Sahi, C., Singh, A, Kumar, K., Blumwald, E., and Grover, A (2006). Salt stress response in rice: genetics, molecular biology, and comparative genomics. Funct. Integr. Genomic. 6, 263-284.

Pubmed: Author and Title

Google Scholar: Author Only Title Only Author and Title

Sakai, H., Lee, S.S., Tanaka, T., Numa, H., Kim, J., Kawahara, Y., Wakimoto, H., Yang, C.-C., Iwamoto, M., Abe, T., Yamada, Y., Muto, A, Inokuchi, H., Ikemura, T., Matsumoto, T., Sasaki, T., and Itoh, T. (2013). Rice Annotation Project Database (RAP-DB): An Integrative and Interactive Database for Rice Genomics. Plant Cell Physiol. 54, e6.

Pubmed: Author and Title

Google Scholar: Author Only Title Only Author and Title

Sandhu, N., Dixit, S., Swamy, B.P.M., Vikram, P., Venkateshwarlu, C., Catolos, M., and Kumar, A (2018). Positive interactions of majoreffect QTLs with genetic background that enhances rice yield under drought. Sci. Rep. 8, 1626.

Pubmed: Author and Title

Google Scholar: Author Only Title Only Author and Title

Schindelin, J., Arganda-Carreras, I., Frise, E., Kaynig, V., Longair, M., Pietzsch, T., Preibisch, S., Rueden, C., Saalfeld, S., and Schmid, B. (2012). Fiji: an open-source platform for biological-image analysis. Nat. Methods 9, 676-682.

Pubmed: Author and Title

Google Scholar: Author Only Title Only Author and Title

Schmidt, R., Schippers, J.H., Mieulet, D., Obata, T., Fernie, AR., Guiderdoni, E., and Mueller-Roeber, B. (2013). MULTIPASS, a rice R2R3-type MYB transcription factor, regulates adaptive growth by integrating multiple hormonal pathways. Plant J. 76, $258-273$.

Pubmed: Author and Title

Google Scholar: Author Only Title Only Author and Title

Schmitz, AJ., Folsom, J.J., Jikamaru, Y., Ronald, P., and Walia, H. (2013). SUB1A-mediated submergence tolerance response in rice involves differential regulation of the brassinosteroid pathway. New Phytol. 198, 1060-1070.

Pubmed: Author and Title

Google Scholar: Author Only Title Only Author and Title

Si, Y., Liu, P., Li, P., and Brutnell, T.P. (2013). Model-based clustering for RNA-seq data. Bioinformatics 30, $197-205$.

Pubmed: Author and Title

Google Scholar: Author Only Title Only Author and Title

Singh, R.K., Gregorio, G.B., and Jain, R.K. (2007). QTL mapping for salinity tolerance in rice. Physiol. Mol. Biol. Plants 13, 87.

Pubmed: Author and Title

Google Scholar: Author Only Title Only Author and Title

Smoot, M.E., Ono, K., Ruscheinski, J., Wang, P.-L., and Ideker, T. (2010). Cytoscape 2.8: new features for data integration and network visualization. Bioinformatics 27, 431-432.

Pubmed: Author and Title

Google Scholar: Author Only Title Only Author and Title

Song, Y., You, J., and Xiong, L. (2009). Characterization of OsIAA1 gene, a member of rice Aux/IAA family involved in auxin and brassinosteroid hormone responses and plant morphogenesis. Plant Mol. Biol. 70, 297-309.

Pubmed: Author and Title

Google Scholar: Author Only Title Only Author and Title

Suzuki, K., Yamaji, N., Costa, A, Okuma, E., Kobayashi, N.I., Kashiwagi, T., Katsuhara, M., Wang, C., Tanoi, K., and Murata, Y. (2016). OsHKT1; 4-mediated $\mathrm{Na}+$ transport in stems contributes to $\mathrm{Na}+$ exclusion from leaf blades of rice at the reproductive growth stage upon salt stress. BMC Plant Biol. 16, 22.

Pubmed: Author and Title

Google Scholar: Author Only Title Only Author and Title

Suzuki, R., and Shimodaira, H. (2006). Pvclust: an R package for assessing the uncertainty in hierarchical clustering. Bioinformatics 22, 1540-1542.

Pubmed: Author and Title

Google Scholar: Author Only Title Only Author and Title

Szabados, L., and Savoure, A (2010). Proline: a multifunctional amino acid. Trends Plant Sci. 15 , 89-97.

Pubmed: Author and Title

Google Scholar: Author Only Title Only Author and Title

Tank, J.G., and Thaker, V.S. (2011). Cyclin dependent kinases and their role in regulation of plant cell cycle. Biol. Plant. 55, 201.

Pubmed: Author and Title 
Google Scholar: Author Only Title Only Author and Title

Tanksley, S.D., and Nelson, J.C. (1996). Advanced backcross QTL analysis: a method for the simultaneous discovery and transfer of valuable QTLs from unadapted germplasm into elite breeding lines. Theor. Appl. Genet. 92, $191-203$.

Pubmed: Author and Title

Google Scholar: Author Only Title Only Author and Title

The UniProt Consortium (2018). UniProt: a worldwide hub of protein knowledge. Nucleic Acids Res. 47, D506-D515.

Pubmed: Author and Title

Google Scholar: Author Only Title Only Author and Title

Thomson, M.J., de Ocampo, M., Egdane, J., Rahman, M.A, Sajise, AG., Adorada, D.L., Tumimbang-Raiz, E., Blumwald, E., Seraj, ZI., Singh, R.K., Gregorio, G.B., and Ismail, AM. (2010). Characterizing the Saltol Quantitative Trait Locus for Salinity Tolerance in Rice. Rice 3, 148-160.

Pubmed: Author and Title

Google Scholar: Author Only Title Only Author and Title

Tyerman, S.D., Munns, R., Fricke, W., Arsova, B., Barkla, B.J., Bose, J., Bramley, H., Byrt, C., Chen, Z, Colmer, T.D., Cuin, T., Day, D.A, Foster, Kylie J., Gilliham, M., Henderson, S.W., Horie, T., Jenkins, C.L.D., Kaiser, B.N., Katsuhara, M., Plett, D., Miklavcic, S.J., Roy, S.J., Rubio, F., Shabala, S., Shelden, M., Soole, K., Taylor, N.L., Tester, M., Watt, M., Wege, S., Wegner, L.H., and Wen, Z (2019). Energy costs of salinity tolerance in crop plants. New Phytol. 221, 25-29.

Pubmed: Author and Title

Google Scholar: Author Only Title Only Author and Title

Ueno, D., Sasaki, A, Yamaji, N., Miyaji, T., Fujii, Y., Takemoto, Y., Moriyama, S., Che, J., Moriyama, Y., Iwasaki, K., and Ma, J.F. (2015). A polarly localized transporter for efficient manganese uptake in rice. Nat. Plants 1, 15170.

Pubmed: Author and Title

Google Scholar: Author Only Title Only Author and Title

Wagner, G.P., and Lynch, V.J. (2010). Evolutionary novelties. Curr Biol. 20, R48-R52.

Pubmed: Author and Title

Google Scholar: Author Only Title Only Author and Title

Walia, H., Wilson, C., Condamine, P., Liu, X., Ismail, AM., Zeng, L., Wanamaker, S.I., Mandal, J., Xu, J., Cui, X., and Close, T.J. (2005). Comparative Transcriptional Profiling of Two Contrasting Rice Genotypes under Salinity Stress during the Vegetative Growth Stage. Plant Physiol. 139, 822-835.

Pubmed: Author and Title

Google Scholar: Author Only Title Only Author and Title

Waziri, A, Kumar, P., and Purty, R. (2016). Saltol QTL and their role in salinity tolerance in rice. Austin J. Biotechnol. Bioeng. 3, 1067.

Pubmed: Author and Title

Google Scholar: Author Only Title Only Author and Title

Wickham, H., and Chang, W. (2008). ggplot2: An implementation of the Grammar of Graphics. R package version 0.7, URL: http://CRAN. R-project. org/package= ggplot2 3.

Pubmed: Author and Title

Google Scholar: Author Only Title Only Author and Title

Xiang, J., Ran, J., Zou, J., Zhou, X., Liu, A, Zhang, X., Peng, Y., Tang, N., Luo, G., and Chen, X. (2013). Heat shock factor OsHsfB2b negatively regulates drought and salt tolerance in rice. Plant Cell Rep. 32, 1795-1806.

Pubmed: Author and Title

Google Scholar: Author Only Title Only Author and Title

Yang, Z, Wu, Y., Li, Y., Ling, H.-Q., and Chu, C. (2009). OsMT1a, a type 1 metallothionein, plays the pivotal role in zinc homeostasis and drought tolerance in rice. Plant Mol. Biol. 70, 219-229.

Pubmed: Author and Title

Google Scholar: Author Only Title Only Author and Title

Yasumoto, A (2020). mytools: Small tools for data analysis.

Yoshida, S., Forno, D.A, and Cock, J.H. (1971). Laboratory manual for physiological studies of rice. (Los Baños, Philippines).

Pubmed: Author and Title

Google Scholar: Author Only Title Only Author and Title

Zhou, G., Xu, Y., Li, J., Yang, L., and Liu, J.-Y. (2006). Molecular analyses of the metallothionein gene family in rice (Oryza sativa L.). BMB Rep. 39, 595-606.

Pubmed: Author and Title

Google Scholar: Author Only Title Only Author and Title

List of Figures 